\title{
Radio Resource Allocation for OFDM-Based Dynamic Spectrum Sharing: Duality Gap and Time Averaging
}

\author{
Mohammad G. Khoshkholgh, Nader Mokari Yamchi, Student Member, IEEE, Keivan Navaie, Senior Member, IEEE, \\ Halim Yanikomeroglu, Senior Member, IEEE, Victor C. M. Leung, Fellow, IEEE, and \\ Kang G. Shin, Life Fellow, IEEE
}

\begin{abstract}
This paper considers radio resource allocation (RRA) in the downlink of an orthogonal frequency division multiple access (OFDM)-based spectrum-sharing network. The objective of RRA is to maximize the average achievable throughput subject to the primary service interference threshold and the secondary service transmit power constraint. RRA is usually implemented based on a time window $T$ over which system parameters are averaged and checked against resource constraints. We use shortterm $(T=1$ time slot) and long-term $(T \gg 1$ slots $)$ averaging as approximations to instantaneous and average constraints, respectively. RRA is also investigated for this system with long-term interference threshold and short-term interference threshold constraints. RRA optimization is a nonconvex optimization problem in which the duality principle is adopted to obtain approximate solutions. The duality gap indicates the degree of approximation in the thus-obtained solution. We prove that the duality gap corresponding to each resource allocation asymptotically decays at least with an exponential rate of $T$. We further show that OFDMA is, asymptotically, the optimal subcarrier assignment. We also propose a practically implementable online power and subcarrier allocation with on-the-fly channel state information measurement. An extensive simulation study has been conducted to verify the theoretically predicted duality gap behavior and to investigate the impact of different system parameters on the secondary service performance. The developed algorithms are also validated to be robust in practical settings and converge fast to theoretical bounds, and thus practically implementable.
\end{abstract}

Index Terms-Radio resource allocation, spectrum sharing, time averaging non-convex optimization, duality gap, interference threshold.

Manuscript received January 5, 2014; revised May 18, 2014 and July 18 2014; accepted August 23, 2014. Date of publication October 1, 2014; date of current version April 21, 2015.

M. G. Khoshkholgh and V. C. M. Leung are with the Department of Electrical and Computer Engineering, The University of British Columbia, Vancouver, BC V6T 1Z4, Canada (e-mail: m.g.khoshkholgh@gmail.com; vleung@ece.ubc.ca).

N. M. Yamchi is with the Department of Electrical and Computer Engineering, Tarbiat Modares University, Tehran 14155-4843, Iran.

K. Navaie is with the School of Electronic and Electrical Engineering, University of Leeds, Leeds LS2 9JT, U.K. (e-mail: keivan.navaie@ieee.org).

H. Yanikomeroglu is with the Department of Systems and Computer Engineering, Carleton University, Ottawa, ON K1S 5B6, Canada.

K. G. Shin is with the Department of Electrical Engineering and Computer Science, University of Michigan, Ann Arbor, MI 48109 USA (e-mail: kgshin@umich.edu).

Color versions of one or more of the figures in this paper are available online at http://ieeexplore.ieee.org.

Digital Object Identifier 10.1109/JSAC.2014.2361080

\section{INTRODUCTION}

$\mathbf{I}$ $\mathrm{N}$ spectrum-sharing networks, secondary users are granted to opportunistically access the un-/under-utilized parts of primary service spectrum by adopting the overlay, underlay or mixed spectrum sharing strategies [1]-[4]. Our focus in this paper is on underlay spectrum sharing [5]. The proposal of several recent technologies such as cognitive femtocell systems and device-to-device (D2D) communications in cellular systems [6]-[8] has prompted vast exploration of the underlay spectrum-sharing.

Radio resource allocation (RRA) has been investigated extensively for spectrum-sharing networks with multiple-input multiple-output, multiple access channel, broadcast channel, or ad hoc paradigms [5], [9]-[12]. Sophisticated techniques such as multi-objective optimization have recently been explored for inspecting the interrelationships among heterogenous objectives such as achievable spectral efficiency and energy/power consumption [13]-[15]. Furthermore, fast and distributed techniques like message-passing and belief-propagation have been considered for devising effective spectrum access protocols and interference coordination among coexisting services [16], [17].

The prime scope of this paper is RRA in multi-carrier dynamic spectrum-sharing systems. The authors of [18] studied the optimal bandwidth and power allocation in FDMA-based cognitive radio, in which constraints on peak/average transmit power and interference thresholds were considered. Random sub-channel allocation was proposed for OFDM-based cognitive radio systems, and its impact on capacity scaling was evaluated in [19]. Assuming a single secondary service OFDM link, an efficient power control algorithm for utilizing active and inactive parts of the spectrum was suggested in [20]. The results were then extended to the multiuser scenario in [21]. A practical subcarrier assignment method was also proposed with moderate computational complexity in [22] where the RRA was made much slower than the speed of fading fluctuations.

On the other hand, orthogonal frequency division multiple access (OFDMA) is commonly accepted as the de facto standard for subcarrier assignment in many practical communication systems like WiMAX due mainly to its inherent simplicity, flexibility and scalability, and is preferred for cognitive radio networks. RRA for OFDMA-based spectrum sharing has been studied broadly. Adopting barrier methods coupled with Newton updates, the authors of [23] developed low 
computational algorithms for implementing fast subcarrier assignment. Power and subcarrier allocation for maximizing ergodic capacity of the secondary service was the subject of the study in [24] under the assumption of unavailability of CSI at the secondary service. The authors of [25] focused on chanceconstrained techniques to deal with CSI uncertainty. Benefits of cooperative communications for boosting the performance of RRA in cognitive radio systems were investigated in [26][29]. High performance can be advertised as a compelling incentive for the secondary services to cooperate with the primary service in relaying parts of the primary service's traffic. Spectral efficiency is improved by setting up the cooperative protocols among the secondary users [27]. Energy-efficiency was scrutinized in [30], helping the recent industrial and academic researchers reduce energy expenditure of the information and communication technology sector. The energy-utility tradeoff for optimal power control and scheduling was also the focus in [26]. Utility maximization in multi-cell cognitive radio networks was studied in [31], and efficient scheduling and power allocation schemes were proposed.

Nevertheless, a number of fundamental issues of RRA in multi-carrier spectrum-sharing systems have not yet been studied thoroughly. For instance, despite the popularity of OFDMA, its optimality still remains questionable. The OFDMA-based RRA problems seen in the literature are inherently non-convex [32], so the solutions have nonzero-in many cases unknown-duality gaps, i.e., the difference between the optimal and sub-optimal solutions (which is 0 for convex problems). Note that a nonzero duality gap will inevitably result in resource misuse. Increasing the number of sub-channels has been recognized as a popular mechanism in mitigating the nonzero duality gap. Assuming instantaneous spectral efficiency as the objective function, evaluation of the duality gap with respect to the number of sub-channels was carried out in [33]-[37]. Their primary conclusion is that the growth of the number of sub-channels speeds the decaying rate of the duality gap, at least by $1 / \sqrt{N}$, where $N$ is the number of subchannels [33], [35]. Unfortunately, it may not always be possible to increase the number of sub-channels because of inherent spectrum shortage, especially in the case of spectrum-sharing. On the other hand, simulations and asymptotic evaluations in [38], [39] reveal that for the case of ergodic capacity, the duality gap is virtually 0 , even with a moderate number of subchannels. This finding is promising as it indicates a practical alternative for reducing the duality gap in spectrum-sharing systems. Nonetheless, achieving the ergodic capacity comes with large decoding delay and high system complexity. Moreover, there is no theoretic/numeric indication for how fast the duality gap dies out. Such information can allow a compromise between the tolerable complexity and the desirable duality gap. To shed lights on the nature of the duality gap versus time, we introduce a time window, denoted by $T$, for adjustable averaging of the objective and resource constraints, where $T=1(T \rightarrow \infty)$ is equivalent to the instantaneous (expected) value of the performance metric. The main questions to be addressed are then: Is time window $T$ beneficial in resource allocation?; how does the duality gap behave with respect to the time window T?; when and under what condition is OFDMA the optimal solution?; how robust are the proposed resource allocation algorithms against practical scenarios?

Analysis of the duality gap behavior as a function of $T$ is of practical importance. One may decide to vary the size of time window, $T$, instead of increasing the number of sub-channels [33], to find an approximation to the optimal solution. This is more pragmatic as it is easier and cheaper to adjust the time window than the number of subcarriers. First, it is a matter of the protocol design, not the system construction, and is inexpensive to implement. Second, according to the analysis in this paper, as $T$ increases, the duality gap gets narrower more quickly than when the number of subcarriers grows. Third, in the case of dynamic spectrum sharing, the available spectrum for the secondary transmissions is probably limited, and hence increasing $T$ is a practical solution to reach the optimal resource usage. In such a case, increasing $T$ will compensate for losses due to small available bandwidth. Finally, in wireless environments, the coherence bandwidth may restrict the gain achievable by increasing the number of subcarriers.

This paper makes the following main contributions:

Analytical evaluation of duality gap behavior as a function of $T$ : We estimate the duality gap for the system under consideration and investigate the impact of time scale of operation on the duality gap. The duality gap is shown to vanish asymptotically with an exponential rate of $T$. Our simulation results also confirm this analytical result.

Optimality of exclusive subcarrier assignment: We prove that with probability 1 , the optimal subcarrier assignment is OFDMA, i.e., each subcarrier can be allocated to at most one user. Since the duality gap is asymptotically 0 , this indicates that OFDMA is the optimal, albeit asymptotically, subcarrier assignment.

Practically deployable resource-allocation algorithms: The RRA algorithms developed in this paper require the availability of all channel realizations in the entire time span of the window for off-line solutions of the RRA problems and the optimal Lagrangian multipliers. Besides, the resource-allocation problems should be solved over a period of $T$ time slots, which may exacerbate computational complexity. Nevertheless, by employing sophisticated online mechanisms, we are able to alleviate the scarcity of non-causal CSI. Thus, the algorithms work efficiently based only on the availability of on-the-fly CSI measurements in the corresponding time slots. Additionally, the complexity of the online method is almost as high as the case in which the resource allocation is solved individually for each time slot. These attributes simplify the implementation and broaden the applicability of the algorithms.

The rest of this paper is organized as follows. Section II presents the system model while Section III investigates RRA with the long-term interference threshold constraint. The problem including the short-term interference threshold constraint is investigated in Section IV. We present the simulation results in Section V and conclude the paper in Section VI.

\section{SYSTEM MOdEL}

Fig. 1 shows a schematic of the system model under consideration. We consider a spectrum-sharing network between the 


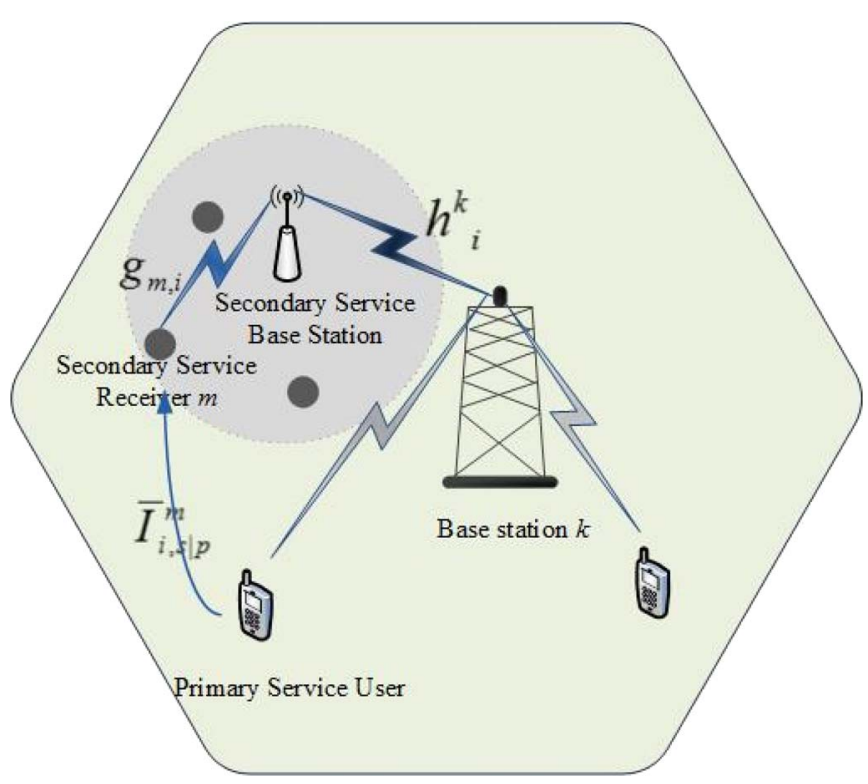

Fig. 1. Schematic diagram of the considered system model in this paper.

primary and secondary services. The primary service is a direct sequence code division multiple access (DS-CDMA)-based cellular network while the secondary service is an OFDMbased cellular network.

Remark 1: Although the analysis in this paper is for DSCDMA/OFDM, our results can be extended to other system settings. For such an extension, optimization problems similar to that in this paper can be formulated and analyzed using the appropriate system model.

We consider a $B-\mathrm{Hz}$ spectrum band that is licensed to the primary service, and the secondary service uses it with an underlay spectrum-sharing mechanism. It is assumed that on this spectrum the primary service communication is uplink while the secondary service communication is downlink. ${ }^{1}$ The secondary service has to keep the imposed interference at the primary service below an a priori assigned interference level referred to as the interference threshold constraint, $Q$. We call this spectrum-sharing scenario DS-CDMA/OFDM [40]. The spectrum is shared over the uplink channel of the primary service only. $^{2}$

For the secondary service, the available spectrum is divided into $N B_{c} \mathrm{~Hz}$ sub-channels, indexed by $i=1,2, \ldots, N$, where $B_{c}$ is assumed equal to the channel coherence bandwidth, so that each sub-channel experiences flat fading. Interference at the secondary service receivers is caused by the primary users' communications. This interference is represented as $\bar{I}_{i, s \mid p}$ which is the aggregated average interference at the secondary service receiver due to the primary service communi-

\footnotetext{
${ }^{1}$ We assume that a fixed portion of the spectrum is allocated to the primary service in the uplink, which will be shared with the secondary service; i.e., the primary network is frequency domain duplexed (FDD), and so is the secondary network.

${ }^{2}$ This is due mainly to the fact that most conventional services are asymmetric, with a lower traffic demand over the uplink than over the downlink. The secondary system should therefore evaluate the interference threshold constraint only at the primary base stations. In practice, this is much simpler than the case of spectrum sharing over the downlink of the primary service, in which the interference threshold constraint should be evaluated at each (mobile) primary users.
}

cations over sub-channel $i$. For subcarrier $i, h_{i}^{k}$ denotes the channel power gain between the secondary BS and the $k$-th primary $\mathrm{BS}$, where $k=1, \ldots, K$, as it is also shown in Fig. 1. In this model, $g_{m, i}$ indicates the instantaneous channel power gain of subcarrier $i$ between the secondary base station (BS) and the secondary service receiver $m, m=1, \ldots, M$. Both $\sqrt{h_{i}^{k}}$ and $\sqrt{g_{m, i}}$ are assumed to be independent and identical Rayleigh random variables. Variable $t$ is used as the time index throughout this paper.

At each time instant, we assume that the full knowledge of the channel power gains, i.e., $h_{i}^{k}$ and $g_{m, i}$, and their relative distributions are made available to the secondary BS, $\forall k, m, i$, $k=1, \ldots, K, m=1, \ldots, M$, and $i=1, \ldots, N$. This assumption is justifiable as in [9], [41]. In practice, $g_{m, i}$ can be estimated by the secondary BS adopting appropriate training sequences and/or through a feedback or reciprocity-based mechanism [42], [43]. Further, the primary service may share its knowledge of $h_{i}^{k} \forall k, i$ with the secondary service, for example, by transmitting a training sequence in the same frequency band. Such an approach has been considered before, e.g., [42]. ${ }^{3}$

\section{OPtimAL RRA With LONG-TERM INTERFERENCE THRESHOLD CONSTRAINT}

Let $\boldsymbol{p}[t]=\left[\boldsymbol{p}_{1}^{\prime}[t], \ldots, \boldsymbol{p}_{M}^{\prime}[t]\right]^{\prime}$ be an $M N \times 1$ secondary users power allocation vector at time instant $t$, where $\boldsymbol{p}_{i}[t]=$ $\left[p_{1, i}[t], \ldots, p_{M, i}[t]\right]^{\prime}$ is an $M$-tuple power-allocation vector for all secondary users on subcarrier $i$. Here, $p_{m, i}[t]$ denotes the power assigned to user $m$ on subcarrier $i$. Let $\gamma_{m, i}[t]$ denote the instantaneous signal to interference-plus-noise ratio (SINR) at the receiver of secondary user $m$ on subcarrier $i$ at time instant $t$. Then, ${ }^{4}$

$$
\gamma_{m, i}[t]=\frac{g_{m, i}[t] p_{m, i}[t]}{\sum_{\hat{m} \neq m} g_{m, i}[t] p_{\hat{m}, i}[t]+\bar{I}_{i, s^{m} \mid p}[t]+\sigma_{m}^{2}}
$$

where $\sigma_{m}^{2}$ is the power spectral density of Additive White Gaussian Noise (AWGN). For a large enough $M$, adopting Central Limit Theorem indicates that the secondary service cochannel interference imposed at the $m$-th secondary user by other secondary transmissions over subcarrier $i$ (i.e., the first term in the denominator in (1)) can be modeled as a Gaussian random process. In (1), $\bar{I}_{i, s^{m} \mid p}[t]$ denotes the interference at the

\footnotetext{
${ }^{3}$ Note that the accurate CSI might not be available in practice. However, since the prime scope of this paper is the evaluation of the duality gap and the investigation of the optimality of OFDMA policy, such an assumption on the availability of accurate CSI is valid. In reality, the inaccuracy of the CSI between the BS and the secondary users degrades the measured signal-to-noise ratio (SNR) due to the self-noise. Besides, when the CSI is inaccurate, the BS may schedule users with a higher data rate than the actual affordable channel capacity causing outages. Moreover, the knowledge of $h_{i}^{k} \forall k, i$ is practically limited to the perturbed CSI values or only the channel distributions. Robust and/or probabilistic constraints could thus be considered for incorporating the spectrum-sharing constraints. In general, it is possible to interpret both cases via long- and/or short-term interference threshold constraints. We leave the impact of inaccuracy of the CSI on the results of this paper as future work.

${ }^{4}$ In such a degraded broadcast channel, the BS implies superposition coding that is optimal, but receivers need to adopt accurate interference cancellation which may not be applicable in practice. Instead of canceling the interference, we assume that the receivers consider the co-channel interference as an additive noise which is a "worst case" scenario.
} 
secondary user $m$ imposed by the primary communications on subcarrier $i$ at time $t$ (see Fig. 1). Time-averaged Achievable Rate (TAR) of the secondary service, $R$ (symbol per second per Hertz), is defined as $R=\frac{1}{T} \sum_{t=1}^{T} \sum_{m=1}^{M} \omega_{m} \sum_{i=1}^{N} \frac{1}{2} \log \left(1+\gamma_{m, i}[t]\right)$, where $T \gg 1$ is the length of averaging time window. Under system ergodicity, for large enough $T$, TAR provides a reasonable approximation of the sum weighted achievable ergodic capacity of the secondary service. The optimal RRA problem is then

$$
\begin{aligned}
& \text { Problem } \mathcal{O}^{T I C}: \\
& R=\max _{\{\boldsymbol{p}[t]\} \in\{\mathcal{P}[t]\}} \frac{1}{T} \sum_{t=1}^{T} \sum_{m=1}^{M} \omega_{m} \sum_{i=1}^{N} \frac{1}{2} \log \left(1+\gamma_{m, i}[t]\right), \\
& \text { s.t. } \quad(T P C),(T I C)
\end{aligned}
$$

in which Time-averaged transmission Power Constraint (TPC) at the secondary BS is $\frac{1}{T} \sum_{t=1}^{T} \sum_{m=1}^{M} \sum_{i=1}^{N} p_{m, i}[t] \leq P_{T}$, where $P_{T}$ (Watts) is the maximum secondary BS average power. The interference threshold constraint at the $k$-th primary BS is managed here through Time-averaged Interference threshold Constraint (TIC) as $\frac{1}{T} \sum_{t=1}^{T} \sum_{m=1}^{M} \sum_{i=1}^{N} h_{i}^{k}[t] p_{m, i}[t] \leq Q_{k}^{L T}, \forall k$, where $Q_{k}^{L T}$ (Watts) is the $k$-th TIC at the primary service receiver. In $\mathcal{O}^{T I C}$, the feasible power allocation set on subcarrier $i$ at time instant $t$ is defined as

$$
\mathcal{P}_{i}[t]=\left\{\boldsymbol{p}_{i}[t] \mid p_{m, i}[t] \geq 0, \forall m\right\} .
$$

Consequently, the feasible power-allocation set at time $t$ is $\mathcal{P}[t]=\mathcal{P}_{1}[t] \times \ldots \times \mathcal{P}_{N}[t]$. Note that $\mathcal{O}^{T I C}$ is a non-convex optimization problem. To solve non-convex optimization problems, the dual decomposition method (e.g., [33]) is utilized to obtain suboptimal solutions. This method is widely used for RRA due to its simplicity as well as the availability of the corresponding effective and quickly converging numerical algorithms.

\section{A. Evaluation of the Duality Gap}

The difference between the obtained solution using dual decomposition and the optimal solution is referred to as the duality gap as $\inf (D(\lambda, \boldsymbol{\mu}))-\sup \left(\mathcal{O}^{T I C}\right)$, where $D(\lambda, \boldsymbol{\mu})$ is the dual function corresponding to $\mathcal{O}^{T I C}$, and $\lambda$ and $\boldsymbol{\mu}=$ $\left[\mu_{1}, \ldots, \mu_{K}\right]^{T}$ are the Lagrangian multipliers corresponding to the constraints in $\mathcal{O}^{T I C}$. Estimating the duality gap indicates how accurately the dual decomposition method solve the resource allocation problem. Hence, several attempts have been made to evaluate the duality gap. For instance, for the special case of $\mathcal{O}^{T I C}$, where $T \rightarrow \infty$, and the power constraint is dominant, i.e., $Q_{k}^{L T}=\infty$, for $k=1, \ldots, K$, the simulation result in [38] indicates that the dual decomposition method yields a solution while satisfying TPC with probability 0.999999 . For a system without spectrum-sharing constraints and when $T=1$, it is shown that for a system with a large number of subcarriers, i.e., $N \rightarrow \infty$, the corresponding duality gap decays to 0 with rate $O\left(\frac{1}{\sqrt{N}}\right)$ [33]-[35].
For problem $\mathcal{O}^{T I C}$ in the following, we show that for almost all practical situations including a finite number of available subcarriers, the duality gap asymptotically approaches 0 with an exponential rate of $T$. As a direct result of this observation, one may decide to increase the time window, $T$, instead of increasing the number of subcarriers to achieve a better approximation to the optimal solution, or even a combination thereof. We first provide the following definition:

Definition: $\varepsilon$-duality gap is defined as $\mathcal{D}(\varepsilon)=\mathbb{P}\{\inf (D(\lambda, \boldsymbol{\mu}))-$ $\left.\sup \left(\mathcal{O}^{T I C}\right)>\varepsilon\right\}$, where $\varepsilon>0$ is a bounded real number.

The following theorem states our main result.

Theorem 1: $\varepsilon$-duality gap corresponding to $\mathcal{O}^{T I C}$ is upperbounded as $\mathcal{D}(\varepsilon) \leq 1-\left(1-e^{-c \varepsilon^{2} T}\right)^{M N T}$. Thus, $\mathcal{D}(\varepsilon)$ approaches 0 by increasing $T \gg 1$ with a decaying rate larger than, or equal to $\left(1-e^{-c \varepsilon^{2} T}\right)^{M N T}$, where $c$ is a constant depending on the system parameters.

Proof: See Appendix A.

Remark 2: The impact of the number of subcarriers on the duality gap is also a matter of interest. As mentioned in Section II, we assume that the bandwidth of each subcarrier is set to be equal to the corresponding channel coherence bandwidth. Therefore, for user $m$, the subchannel power gains, $g_{m, i}, \forall i$, are independent. Increasing the number of subcarriers results in highly correlated subchannels, thus requiring a more complex approach to estimating $\mathcal{D}(\varepsilon)$. For the simple case of $T=1$, the authors of [35] proposed an approach to estimating the duality gap as a function of the number of subcarriers.

Remark 3: Although Theorem 1 holds for the case when channel power gains are Rayleigh, a similar behavior is also expected in other fading environments. In such a case, the only modification is the rate at which the duality gap vanishes; more specifically, in the proof of Theorem 1 we need to modify

$$
\begin{aligned}
& \mathbb{P}\left\{\sup \{r \mid \tilde{\mathcal{P}}\}>\frac{T \varepsilon}{K+2}\right\} \\
& \leq 1-\left(1-\bar{F}_{g_{m, i}}\left(\frac{e^{\frac{T \varepsilon}{N M(K+2)}}-1}{p_{\max }} \min _{m, i}\left\{\mu_{m, i} \bar{I}_{i, s^{m} \mid p}\right\}\right)\right)^{M N},
\end{aligned}
$$

where $\bar{F}_{g_{m, i}}($.$) is the complementary probability distribution$ function of random variable $g_{m, i}$. Note that depending on the tail of the random variable $g_{m, i}$, the rate of reduction might be either faster or slower than exponential.

\section{B. Dual Solution}

The dual problem corresponding to Problem $\mathcal{O}^{T I C}$ is defined as $D^{*}=\min _{\lambda \geq 0, \boldsymbol{\mu} \geq 0} D(\lambda, \boldsymbol{\mu})$. The corresponding dual function for $\mathcal{O}^{T I C}$ is

$$
\begin{gathered}
D(\lambda, \boldsymbol{\mu})=\max _{\{\boldsymbol{p}[t]\} \in\{\mathcal{P}[t]\}} \frac{1}{T} \sum_{t, m, i} \frac{\omega_{m}}{2} \log \left(1+\gamma_{m, i}[t]\right) \\
+\lambda\left(P_{T}-\sum_{t, m, i} \frac{p_{m, i}[t]}{T}\right)+\sum_{k=1}^{K} \mu_{k}\left(Q_{k}^{L T}-\sum_{t, m, i} \frac{h_{i}^{k}[t] p_{m, i}[t]}{T}\right) \\
=\lambda P_{T}+\sum_{k=1}^{K} \mu_{k} Q_{k}^{L T}+\frac{1}{T} \sum_{t=1}^{T} D(\lambda, \boldsymbol{\mu})[t]
\end{gathered}
$$


This decomposes the problem into $T$ decoupled optimization problems, where for time instant $t$,

$$
\begin{aligned}
D(\lambda, \boldsymbol{\mu})[t]=\sum_{i=1}^{N} \max _{\boldsymbol{p}_{i}[t] \in \mathcal{P}_{i}[t]} & \sum_{m=1}^{M}\left(\frac{\omega_{m}}{2} \log \left(1+\gamma_{m, i}[t]\right)\right. \\
& \left.-\left(\lambda+\sum_{k=1}^{K} \mu_{k} h_{i}^{k}[t]\right) p_{m, i}[t]\right) .
\end{aligned}
$$

Therefore, the primal problem is decomposed further into $N T$ decoupled optimization problems. For subcarrier $i$, at time instant $t$, the corresponding optimization problem is

Problem $\mathcal{O}^{i}$ :

$$
\begin{aligned}
& \max _{p_{m, i}[t] \geq 0, \forall m} \sum_{m=1}^{M}-\left(\lambda+\sum_{k=1}^{K} \mu_{k} h_{i}^{k}[t]\right) p_{m, i}[t] \\
& \times \frac{\omega_{m}}{2} \log \left(1+\frac{g_{m, i}[t] p_{m, i}[t]}{\sum_{\hat{m} \neq m} g_{m, i}[t] p_{\hat{m}, i}[t]+\bar{I}_{i, s^{m} \mid p}[t]+\sigma_{m}^{2}}\right) .
\end{aligned}
$$

Proposition 1: In the optimal solution of $\mathcal{O}^{i}$, for each time $t$ and specific subcarrier $i$, at most one user is active. The active user on subcarrier $i$ is

$$
m_{i}^{*}[t]=\arg \max _{m=1, \ldots, M} \omega_{m} \frac{g_{m, i}[t]}{\bar{I}_{i, s^{m} \mid p}[t]+\sigma_{m}^{2}} .
$$

Proof: We first rewrite the objective function of $\mathcal{O}^{i}$ as

$$
\begin{aligned}
\sum_{m=1}^{M} & \frac{\omega_{m}}{2} \log \left(\sum_{\hat{m}=1}^{M} g_{m, i}[t] p_{\hat{m}, i}[t]+\bar{I}_{i, s^{m} \mid p}[t]+\sigma_{m}^{2}\right) \\
& -\sum_{m=1}^{M}\left(\lambda+\sum_{k=1}^{K} \mu_{k} h_{i}^{k}[t]\right) p_{m, i}[t] \\
& -\sum_{m=1}^{M} \frac{\omega_{m}}{2} \log \left(\sum_{\hat{m} \neq m} g_{m, i}[t] p_{\hat{m}, i}[t]+\bar{I}_{i, s^{m} \mid p}[t]+\sigma_{m}^{2}\right) .
\end{aligned}
$$

For user $m_{1}$, the Karush-Kuhn-Tucker (KKT) conditions [32], which provide sufficient conditions for the optimal solution of $\mathcal{O}^{i}$, are

$$
\begin{array}{r}
\sum_{m=1}^{M} \frac{\frac{\omega_{m}}{2 \ln 2}}{\frac{\bar{I}_{i, s} m_{\mid p}[t]+\sigma_{m}^{2}}{g_{m, i}[t]}+\sum_{\hat{m}=1}^{M} p_{\hat{m}, i}^{*}[t]}-\left(\lambda+\sum_{k=1}^{K} \mu_{k} h_{i}^{k}[t]\right) \\
=\sum_{m \neq m_{1}} \frac{\frac{\omega_{m}}{2 \ln 2}}{\frac{\bar{I}_{i, s} m_{p p}[t]+\sigma_{m}^{2}}{g_{m, i}[t]}+\sum_{\hat{m} \neq m} p_{\hat{m}, i}^{*}[t]}-\delta_{m_{1}, i}^{*}[t],
\end{array}
$$

$\delta_{m_{1}, i}^{*}[t] p_{m_{1}, i}^{*}[t]=0, \quad \delta_{m_{1}, i}^{*}[t] \geq 0, \quad$ and $p_{m_{1}, i}^{*}[t] \geq 0$ where $p_{m_{1}, i}^{*}[t]$ is the optimal power allocated to user $m_{1}$ on subcarrier $i$ at time instant $t$. Furthermore, $\delta_{m, i}^{*}[t]$ are Lagrange multipliers corresponding to the constraints in $\mathcal{O}^{i}$. Now, we assume that two users, say $m_{1}=1$ and $m_{2}=2$, are active at time instant $t$, i.e., $p_{1, i}^{*}[t]>0$, and $p_{2, i}^{*}[t]>0$. Thus, we have $\delta_{1, i}^{*}[t]=0$, $\delta_{2, i}^{*}[t]=0$. Let us denote

$$
\Psi=\sum_{m=1}^{M} \frac{\frac{\omega_{m}}{2 \ln 2}}{\frac{\left.\bar{I}_{i, s}\right|_{\mid p}[t]+\sigma_{m}^{2}}{g_{m, i}[t]}+p_{1, i}^{*}[t]+p_{2, i}^{*}[t]} .
$$

We write (8) for both users, $m_{1}$ and $m_{2}$, respectively, as:

$$
\begin{aligned}
& \frac{\frac{\omega_{2}}{2 \ln 2}}{\frac{M}{\frac{\bar{I}_{i, s^{2} \mid p}[t]+\sigma_{2}^{2}}{g_{2, i}[t]}+p_{1, i}^{*}[t]}+\sum_{m=3}^{M} \frac{\frac{\omega_{m}}{2 \ln 2}}{\frac{\bar{I}_{i, s} m \mid p}{g_{m, i}[t]+\sigma_{m}^{2}}}+p_{1, i}^{*}[t]+p_{2, i}^{*}[t]} \\
& =\Psi-\left(\lambda+\sum_{k=1}^{K} \mu_{k} h_{i}^{k}[t]\right) \text {, }
\end{aligned}
$$

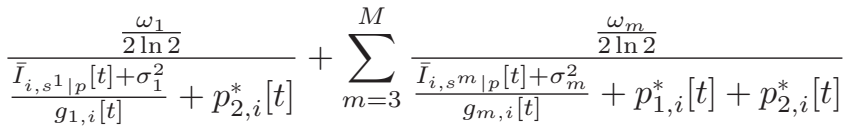

$$
\begin{aligned}
& =\Psi-\left(\lambda+\sum_{k=1}^{K} \mu_{k} h_{i}^{k}[t]\right) \text {. }
\end{aligned}
$$

After some straightforward manipulations, (10) and (11), respectively, reduce to

$$
\begin{gathered}
\frac{\frac{\omega_{1}}{2 \ln 2}}{\frac{\left.\bar{I}_{i, s}|p| t\right]+\sigma_{1}^{2}}{g_{1, i}[t]}+p_{1, i}^{*}[t]+p_{2, i}^{*}[t]}+\frac{\frac{\omega_{2}}{2 \ln 2}}{\frac{\omega_{i, s^{2} \mid p}[t]+\sigma_{2}^{2}}{g_{2, i}[t]}+p_{1, i}^{*}[t]+p_{2, i}^{*}[t]} \\
-\frac{\frac{\omega_{2}}{2 \ln 2}}{\frac{\bar{I}_{i, s^{2} \mid p}[t]+\sigma_{2}^{2}}{g_{2, i}[t]}+p_{1, i}^{*}[t]}=\left(\lambda+\sum_{k=1}^{K} \mu_{k} h_{i}^{k}[t]\right), \\
\frac{\frac{\omega_{1}}{2 \ln 2}}{\frac{\bar{I}_{i, s^{1} \mid p}[t]+\sigma_{1}^{2}}{g_{1, i}[t]}+p_{1, i}^{*}[t]+p_{2, i}^{*}[t]}+\frac{\frac{\omega_{2}}{2 \ln 2}}{\frac{\bar{I}_{i, s^{2} \mid p}[t]+\sigma_{2}^{2}}{g_{2, i}[t]}+p_{1, i}^{*}[t]+p_{2, i}^{*}[t]} \\
-\frac{\frac{\omega_{1}}{2 \ln 2}}{\frac{\bar{I}_{i_{s} s^{1} \mid p}[t]+\sigma_{1}^{2}}{g_{1, i}[t]}+p_{2, i}^{*}[t]}=\left(\lambda+\sum_{k=1}^{K} \mu_{k} h_{i}^{k}[t]\right) .
\end{gathered}
$$

These two equations finally imply that

$$
\frac{\frac{\omega_{2}}{2 \ln 2}}{\frac{\bar{I}_{i, s^{2} \mid p}[t]+\sigma_{2}^{2}}{g_{2, i}[t]}+p_{1, i}^{*}[t]}=\frac{\frac{\omega_{1}}{2 \ln 2}}{\frac{\bar{I}_{i, s^{1} \mid p}[t]+\sigma_{1}^{2}}{g_{1, i}[t]}+p_{2, i}^{*}[t]} .
$$

Note that all involved channel power gains including $g_{m, i} \mathrm{~S}$ are independent across different users on each subcarrier $i$. Further, fading distributions are continuous, so (14) holds with probability 0 , i.e.,

$$
\mathbb{P}\left\{\frac{\frac{\omega_{2}}{2 \ln 2}}{\frac{\bar{I}_{i, s^{2} \mid p}[t]+\sigma_{2}^{2}}{g_{2, i}[t]}+p_{1, i}^{*}[t]}=\frac{\frac{\omega_{1}}{2 \ln 2}}{\frac{\bar{I}_{i, s^{1} \mid p}[t]+\sigma_{1}^{2}}{g_{1, i}[t]}+p_{2, i}^{*}[t]}\right\}=0 .
$$

It is also straightforward to prove that the above statement is valid if more than 2 users are assumed to be active. Therefore, the feasible power set $\dot{\mathcal{P}}[t]$ defined as $\dot{\mathcal{P}}[t]=\dot{\mathcal{P}}_{1}[t] \times \ldots \times$ $\dot{\mathcal{P}}_{N}[t]$, where

$$
\dot{\mathcal{P}}_{i}[t]=\left\{\boldsymbol{p}_{i}[t] \in \mathbb{R}_{+}^{M} \mid p_{m, i}[t] p_{\hat{m}, i}[t]=0, \forall m \neq \hat{m}\right\},
$$

forms the feasible power-allocation set of the dual optimization problem. As shown above, the feasible power set meets the OFDMA constraint, i.e., at each time instant $t$ at most one user, 
say $m_{1}=1$, is active and the others are silent. In this case, (8) is simplified to

$$
\begin{aligned}
& \sum_{m=1}^{M} \frac{\frac{\omega_{m}}{2 \ln 2}}{\frac{\left.\bar{i}_{i, s}\right|_{p}[t]+\sigma_{m}^{2}}{g_{m, i}[t]}+p_{1, i}^{*}[t]}-\sum_{m=2}^{M} \frac{\frac{\omega_{m}}{2 \ln 2}}{\frac{\bar{I}_{i, s} m_{\mid p}[t]+\sigma_{m}^{2}}{g_{m, i}[t]}+p_{1, i}^{*}[t]} \\
& =\left(\lambda+\sum_{k=1}^{K} \mu_{k} h_{i}^{k}[t]\right),
\end{aligned}
$$

or equivalently,

$$
\left(\lambda+\sum_{k=1}^{K} \mu_{k} h_{i}^{k}[t]\right)=\frac{\frac{\omega_{1}}{2 \ln 2}}{\frac{\bar{I}_{i, s} \mid p[t]+\sigma_{1}^{2}}{g_{1, i}[t]}+p_{1, i}^{*}[t]},
$$

which results in the following well known water-filling power allocation

$$
p_{1, i}^{*}[t]=\left(\frac{\omega_{1}}{2 \ln 2\left(\lambda+\sum_{k=1}^{K} \mu_{k} h_{i}^{k}[t]\right)}-\frac{\bar{I}_{i, s^{1} \mid p}[t]+\sigma_{1}^{2}}{g_{1, i}[t]}\right)^{+} .
$$

For users $m_{2} \neq m_{1}$, on the other hand, (8) indicates

$$
\begin{array}{r}
\sum_{m=1}^{M} \frac{\frac{\omega_{m}}{2 \ln 2}}{\frac{\bar{I}_{i, s} m \mid p}{g_{m, i}[t]+\sigma_{m}^{2}}+p_{1, i}^{*}[t]}-\sum_{m=2, m \neq m_{2}}^{M} \frac{\frac{\omega_{m}}{2 \ln 2}}{\frac{\bar{I}_{i, s} m_{\mid p}[t]+\sigma_{m}^{2}}{g_{m, i}[t]}+p_{1, i}^{*}[t]} \\
-\frac{\frac{\omega_{1}}{2 \ln 2}}{\frac{\bar{I}_{i, s^{1} \mid p}[t]+\sigma_{1}^{2}}{g_{1, i}[t]}}=\left(\lambda+\sum_{k=1}^{K} \mu_{k} h_{i}^{k}[t]\right)-\delta_{m_{2}, i}^{*}[t], \quad \text { (19) }
\end{array}
$$

which simplifies to

$$
\begin{aligned}
& \frac{\frac{\omega_{1}}{2 \ln 2}}{\frac{\overline{\bar{I}_{i, s^{1} \mid p}}[t]+\sigma_{1}^{2}}{g_{1, i}[t]}+p_{1, i}^{*}[t]}-\frac{\frac{\omega_{1}}{2 \ln 2}}{\frac{\bar{I}_{i, s^{1} \mid p}[t]+\sigma_{1}^{2}}{g_{1, i}[t]}}+\frac{\frac{\omega_{m_{2}}}{2 \ln 2}}{\frac{\bar{I}_{i, s} m_{2 \mid p}[t]+\sigma_{m_{2}}^{2}}{g_{m_{2}, i}[t]}+p_{1, i}^{*}[t]} \\
& =\left(\lambda+\sum_{k=1}^{K} \mu_{k} h_{i}^{k}[t]\right)-\delta_{m_{2}, i}^{*}[t] .
\end{aligned}
$$

Substituting from (17), we then solve the above equation for $\delta_{m_{2}, i}^{*}[t]$ as

$$
\delta_{m_{2}, i}^{*}[t]=\frac{\frac{\omega_{1}}{2 \ln 2}}{\frac{\bar{I}_{i, s} \mid p}{g_{1, i}[t]}[t]+\sigma_{1}^{2}}-\frac{\frac{\omega_{m_{2}}}{2 \ln 2}}{\frac{\bar{I}_{i, s} m_{2 \mid p}[t]+\sigma_{m_{2}}^{2}}{g_{m_{2}, i}[t]}+p_{1, i}^{*}[t]}>0 .
$$

Substituting $p_{1, i}^{*}[t]$ from (18), (21) reduces to

$$
\begin{aligned}
& \forall m_{2} \neq 1: \frac{g_{1, i}[t]}{\bar{I}_{i, s^{1} \mid p}[t]+\sigma_{1}^{2}} \frac{\bar{I}_{i, s^{m_{2} \mid p}}[t]+\sigma_{1}^{2}}{g_{m_{2}, i}[t]} \\
& +\left(\frac{g_{1, i}[t]}{\bar{I}_{i, s^{1} \mid p}[t]+\sigma_{1}^{2}} \frac{\omega_{1}}{\left(\lambda+\sum_{k=1}^{K} \mu_{k} h_{i}^{k}[t]\right)}-1\right)^{+}>\frac{\omega_{m_{2}}}{\omega_{1}} .
\end{aligned}
$$

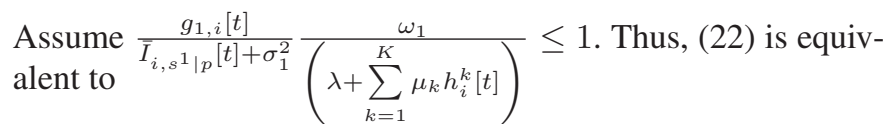

$$
\omega_{1} \frac{g_{1, i}[t]}{\bar{I}_{i, s^{1} \mid p}[t]+\sigma_{1}^{2}}>\omega_{m_{2}} \frac{g_{m_{2}, i}[t]}{\bar{I}_{i, s^{m_{2}} \mid p}[t]+\sigma_{1}^{2}} \quad \forall m_{2} \neq 1 .
$$

On the other hand, if

$$
\frac{g_{1, i}[t]}{\bar{I}_{i, s^{1} \mid p}[t]+\sigma_{1}^{2}} \frac{\omega_{1}}{\left(\lambda+\sum_{k=1}^{K} \mu_{k} h_{i}^{k}[t]\right)}>1,
$$

is valid, then (22) reduces to

$$
\begin{aligned}
& \frac{g_{m_{2}, i}[t]}{\bar{I}_{i, s^{m_{2} \mid p}}[t]+\sigma_{1}^{2}}+\frac{\omega_{1}}{\left(\lambda+\sum_{k=1}^{K} \mu_{k} h_{i}^{k}[t]\right)} \\
& >\left(\frac{\omega_{m_{2}}}{\omega_{1}}+1\right) \frac{g_{1, i}[t]}{\bar{I}_{i, s^{1} \mid p}[t]+\sigma_{1}^{2}} \quad \forall m_{2} \neq 1 .
\end{aligned}
$$

Taking this and (24) into account, it is then straightforward to show that (23) is again valid. This finally proves the result in (6).

Remark 4: For the case of $T=1$ and $Q_{k}^{L T}=\infty, k=$ $1, \ldots, K$ and Quadratic Amplitude Modulation (QAM) in [44], the optimal subcarrier assignment is shown to be OFDMA. For the general case of Shannon capacity formula and when $\omega_{m}=$ 1 , this is also shown based on the quasi-convex optimization properties in [45]. The result of Proposition 1 is more general and based on dual solution.

Remark 5: If OFDMA was part of the formulation of RRA, one could establish that $D(\lambda, \boldsymbol{\mu})[t]$ in (5) is

$$
\begin{aligned}
& \left.D(\lambda, \boldsymbol{\mu})[t]=\sum_{i=1}^{N} \max _{m=1, \ldots, M}-\left(\lambda+\sum_{k=1}^{K} \mu_{k} h_{i}^{k}[t]\right) p_{m, i}[t]\right) \\
& \times \max _{p_{\hat{m}, i}[t] \geq 0}\left(\frac{\omega_{m}}{2} \log \left(1+\frac{g_{m, i}[t] p_{m, i}[t]}{\bar{I}_{i, s^{m} \mid p}[t]+\sigma_{m}^{2}}\right),\right.
\end{aligned}
$$

which admits the water-filling power allocation

$p_{m, i}^{*}[t]=\left(\frac{\omega_{m}}{2 \ln 2\left(\lambda+\sum_{k=1}^{K} \mu_{k} h_{i}^{k}[t]\right)}-\frac{\bar{I}_{i, s^{m} \mid p}[t]+\sigma_{m}^{2}}{g_{m, i}[t]}\right)^{+}$,

and subcarrier assignment $m_{i}^{*}[t]$ is

$$
\arg \max _{m} \frac{\omega_{m}}{2 \ln 2} \ln \left(\frac{\omega_{m}}{2 \ln 2 A_{m}}\right)^{+}-\left(1-\frac{2 \ln 2}{\omega_{m}} A_{m}\right)^{+}
$$

where $A_{m}=\frac{\lambda+\sum_{k=1}^{K} \mu_{k} h_{i}^{k}[t]}{g_{m, i}[t]\left(\bar{I}_{i, s^{m} \mid p}[t]+\sigma_{m}^{2}\right)^{-1}}$. Compared to this, Proposition 1 explicitly highlights the role of $\omega_{m}$ and channel power gains in subcarrier assignment that is implicit and difficult to grasp in (28). These attributes can shed lights on the fairness in resource allocation. 
Remark 6: It is worth pointing out the fundamental distinction between $\mathcal{O}^{i}$ and the counterpart problem in the Interference Channel. According to (1), the system model here comprises one sender and $M$ receivers. The link, say $g_{m, i}$, that is used for conveying information to user $m$ carries the interfering data to this user as well. On the contrary, there are $M$ transmitters and $M$ receivers in the interference channel, so each transmitter in essence has $M-1$ cross-talk links to the others, which may not be the same as the link to the intended receiver. Thus, the link between a transmitter and its intended receiver only conveys the information, not the interfering data. In this regards, Proposition 1 does not depend on any assumptions commonly seen in the literature of interference channel on the particular relationships between direct and cross-talk gains for deriving the capacity bound and/or power allocation [46], [47].

Proposition 1 shows that OFDMA is the optimal subcarrier allocation for TPC/TIC. The optimal power allocation is then obtained as $p_{m, i}^{*}[t]=p_{m_{i}^{*}[t], i}^{*}[t] 1_{m=m_{i}^{*}[t]}$. We also need to obtain the optimal Lagrangian multipliers, which can be done by adopting known convex optimization algorithms, such as Ellipsoid method [32]. The thus-obtained power allocation is then upgraded by substituting $\lambda^{*}$ and $\boldsymbol{\mu}^{*}$ in $p_{m, i}^{*}[t]=$ $p_{m_{i}^{*}[t], i}^{*}[t] 1_{m=m_{i}^{*}[t]}$. A sub-gradient required for such an upgrade is

$$
\begin{gathered}
{\left[P_{T}-\frac{\sum_{t, m, i} p_{m, i}^{*}[t]}{T}, Q_{1}^{L T}-\frac{\sum_{t, m, i} h_{i}^{1}[t] p_{m, i}^{*}[t]}{T},\right.} \\
\left.\ldots, Q_{K}^{L T}-\frac{\sum_{t, m, i} h_{i}^{K}[t] p_{m, i}^{*}[t]}{T}\right]^{\prime}
\end{gathered}
$$

Note that KKT conditions in (8) are only sufficient since Problem $\mathcal{O}^{i}$ is non-convex. As to Theorem 1, we already know that for a sufficiently large $T$, the duality gap is almost 0 , implying that OFDMA is asymptotically the optimal subcarrier assignment.

When the primary service uses multi-carrier technology, we get the same Lagrangian function, perhaps with greater Lagrangian multipliers. The results of Theorem 1 and Proposition 1 remain valid.

\section{System Causality}

In the above dual decomposition discussed, to update the Lagrangian multipliers, at the beginning of a time window, we need all channel realizations within the entire time window (i.e., off-line update). This is impractical in a causal system. Theoretically, one can assume that a genie agent provides the required knowledge. In practice, however, we need a very small duality gap, thus requiring a large $T$ as shown in Theorem 1 . If $T$ is large enough, then this issue can be handled by using the ergodicity of wireless channels. Due to the ergodicity in such a case, with a very high level of accuracy, channel realizations required for updating the Lagrangian multipliers can be achieved by using the expected values instead of the arithmetic average over the entire time window $T$. Using large enough channel realizations, RRA calculates the Lagrangian multipliers before the start of time window by many artificially generated channel realizations according to the given pdfs. In each time slot (within the window), the resources are allocated by substituting the available CSI of the upcoming time slot in the derived formulations for subcarrier assignment and transmit power allocation.

Another way to tackle the non-causality issue is to adopt the stochastic gradient decent method (online update) in [48], [49]. To utilize this method, however, we need to check whether Lemma 3 and Theorem 2 in [49] hold for the system under study. If they do, then the updated Lagrangian multipliers using this method are the same as those in a non-causal system where a priori knowledge within the entire window is available. The simulation results presented in Section V indicate that even for a moderate value of $T$, e.g., $T=300$, online updated Lagrangian multipliers quickly converge to their optimal values obtained from off-line updates, thereby making the developed algorithms implementable in practice. Note that this does not require the ergodicity assumption as in [48], [49].

\section{OPTIMAL RRA With SHORT-TERM INTERFERENCE THRESHOLD CONSTRAINT}

In the previous section we have investigated the scenario with TIC. We have proved that OFDMA is an asymptotically optimal subcarrier policy. However, the primary service might, in practice, be sensitive to peak interference imposed by the secondary service. In this section we confirm that the theory developed in the previous section is also valid for this case.

\section{A. Problem Formulation and Duality Gap}

Let us consider the following RRA problem: Problem $\mathcal{O}^{I C}$ :

$$
\begin{aligned}
& R=\max _{\{\boldsymbol{p}[t]\} \in\{\mathcal{P}[t]\}} \frac{1}{T} \sum_{t=1}^{T} \sum_{m=1}^{M} \omega_{m} \sum_{i=1}^{N} \frac{1}{2} \log \left(1+\gamma_{m, i}[t]\right), \\
& \text { s.t. }(T P C),(I C)
\end{aligned}
$$

where IC is the interference threshold constraint and ensures that the allocated power at the secondary BS must satisfy: $\sum_{m=1}^{M} \sum_{i=1}^{N} h_{i}^{k}[t] p_{m, i}[t] \leq Q_{k}^{S T}, \forall k, \quad \forall t$, where $Q_{k}^{S T}$

is IC. Following the same line of argument discussed in Section III, we prove the main result of this section in the following theorem.

Theorem 2: The $\varepsilon$-duality gap corresponding to $\mathcal{O}^{I C}$ is upper-bounded by

$$
\mathcal{D}(\varepsilon) \leq 1-\left[1-d\left[\frac{1}{e^{c^{\prime} \varepsilon T}+a}-\frac{1}{e^{c^{\prime} \varepsilon T}+b}\right]\right]^{M N^{2} T},
$$

where $c^{\prime}, a, b>a$, and $d$ depend on the system parameters. Then, $\mathcal{D}(\varepsilon)$ approaches 0 by increasing $T \gg 1$ with a decaying rate larger than, or equal to $\left(1-e^{c^{\prime 2} \varepsilon^{2} T^{2}}\right)^{M N^{2} T}$. 
Proof: See Appendix C.

Remark 7: Comparison of Theorem 1 and Theorem 3 reveals that the rate of vanishing the duality gap with TPC/IC combination is higher than that in TPC/TIC combination.

\section{B. Dual Solution}

In this section, we obtain the optimal power and subcarrier allocation for $\mathcal{O}^{I C}$. The dual problem is formulated as $D^{*}=$ $\min _{\lambda \geq 0} D(\lambda)$, where $D(\lambda)$ is the dual function obtained by relaxing the TPC constraint as:

$$
\begin{aligned}
D(\lambda) & =\max _{\{\boldsymbol{p}[t]\} \in\{\mathcal{Q}[t]\}} \frac{1}{T} \sum_{t, m, i} \frac{\omega_{m}}{2} \log \left(1+\gamma_{m, i}[t]\right) \\
& +\lambda\left(P_{T}-\frac{1}{T} \sum_{t, m, i} p_{m, i}[t]\right)=\lambda P_{T}+\frac{1}{T} \sum_{t=1}^{T} D(\lambda)[t] .
\end{aligned}
$$

The dual function is then decomposed for different time instants $t$ as

Problem $\mathcal{O}^{t}$ :

$$
\max _{\boldsymbol{p}[t] \in \mathcal{P}[t], \text { s.t. }(I C)} \sum_{m, i}\left(\frac{\omega_{m}}{2} \log \left(1+\gamma_{m, i}[t]\right)-\lambda p_{m, i}[t]\right) .
$$

The dual function corresponding to $\mathcal{O}^{t}$ is then formulated as $L^{*}[t]=\min _{\boldsymbol{\mu}[t] \geq 0} L(\lambda, \boldsymbol{\mu}[t])[t]$, where $\boldsymbol{\mu}[t]$ represents the Lagrangian vector corresponding to the IC constraint. The dual function $L(\lambda, \boldsymbol{\mu}[t])[t]$ is similarly defined for each $t$ as $L(\lambda, \boldsymbol{\mu}[t])[t]=\sum_{k=1}^{K} \mu_{k}[t] Q_{k}^{L T}+\sum_{i=1}^{N} L_{i}(\lambda, \boldsymbol{\mu}[t])[t]$, where the dual function for given subcarrier and time instant $t$, $L_{i}(\lambda, \boldsymbol{\mu}[t])[t]$, is

$$
\begin{aligned}
L_{i}(\lambda, \boldsymbol{\mu}[t])[t]=\max _{\boldsymbol{p}_{i}[t] \in \mathcal{P}_{i}[t]} \sum_{m=1}^{M} & \left(\frac{\omega_{m}}{2} \log \left(1+\gamma_{m, i}[t]\right)\right. \\
& \left.-\left(\lambda+\sum_{k=1}^{K} \mu_{k}[t] h_{i}^{k}[t]\right) p_{m, i}[t]\right) .
\end{aligned}
$$

By applying the KKT optimality conditions and following the same line of argument for TPC/TIC as in the proof of Proposition 1, we can show that in TPC/IC, OFDMA is also the optimal subcarrier assignment. Therefore, the feasible power allocation set on subcarrier $i$ is $\dot{\mathcal{P}}_{i}[t]$. Consequently,

$$
\begin{aligned}
L_{i}(\lambda, \boldsymbol{\mu}[t])[t]=\max _{m=1, \ldots, M} \max _{p_{m, i} \geq 0}\left(\frac{\omega_{m}}{2} \log \left(1+\gamma_{m, i}[t]\right)\right. \\
\left.-\left(\lambda+\sum_{k=1}^{K} \mu_{k}[t] h_{i}^{k}[t]\right) p_{m, i}[t]\right),
\end{aligned}
$$

where the optimal power $p_{m, i}^{*}[t]$ for the inner optimization is

$$
\left(\frac{\omega_{m}}{2 \ln 2\left(\lambda+\sum_{k=1}^{K} \mu_{k}[t] h_{i}^{k}[t]\right)}-\frac{\bar{I}_{i, s^{m} \mid p}[t]+\sigma_{m}^{2}}{g_{m, i}[t]}\right)^{+} \text {. }
$$

Furthermore, the secondary user selected for transmission on subcarrier $i$ at time instant $t, m_{i}^{*}[t]$, is

$$
m_{i}^{*}[t]=\arg \max _{m=1, \ldots, M} \omega_{m} \frac{g_{m, i}[t]}{\bar{I}_{i, s^{m} \mid p}[t]+\sigma_{m}^{2}} .
$$

For identical $\omega_{m} \mathrm{~s}$, user $m_{i}^{*}[t]$ is chosen such that

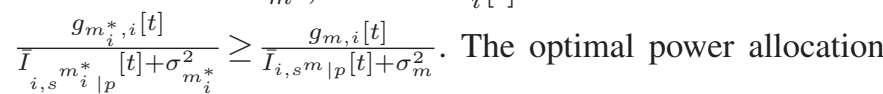
in case of OFDMA is then obtained as $p_{m, i}^{*}[t]=$ $p_{m_{i}^{*}[t], i}^{*}[t] 1_{m=m_{i}^{*}[t]}$.

The optimal Lagrangian vector $\boldsymbol{\mu}^{*}[t]$ is then obtained for each time instant $t$ by utilizing standard solution algorithms for the convex optimization problem, such as the Ellipsoid method. Given the optimal power allocation and subcarrier assignment, the rest is to minimize the dual function $D(\lambda)$ and obtain the optimal Lagrangian multiplier $\lambda^{*}$.

Remark 8: The computational complexity of the optimization problems $\mathcal{O}^{T I C}$ and $\mathcal{O}^{I C}$, respectively, are $\mathcal{O}(T M N(K+$ $\left.1)^{2}\right)$ and $\mathcal{O}\left(T M N(T K+1)^{2}\right)$. Compared to a resource allocation policy whereby the optimization problem $\mathcal{O}^{T I C}$, for example, has to be solved individually in each time slot, which requires the computational complexity $T \mathcal{O}\left(M N(K+1)^{2}\right)$, the computational complexity remains almost the same. However, TPC/IC combination will impose much higher computational complexity than this approach.

\section{Simulations Results}

In this section numerical results are provided to corroborate the analysis. In our numerical study we mainly focus on evaluation of the duality gap, the impact of system parameters on the duality gap and TAR, and finally, causality. While the choices of system parameters do not necessarily adhere to a specific standard, the ranges are wide enough to cover different scenarios. We set noise power 1 Watt for all users across all subcarriers. In the simulations the value considered for interference threshold (both IC and TIC) is scaled with respect to the background noise power. We set coherence bandwidth such that the total available bandwidth is divided into $N=16$ flat fading sub-channels.

\section{A. Evaluation of Duality Gap}

Thus far, we have provided upper-bounds on the optimal value of TAR with TPC/TIC and TPC/IC combinations adopting dual decomposition techniques, and proved that these upper-bounds exponentially converge to the globally optimal values. Since for both TPC/TIC and TPC/IC combinations, the exact duality gap value might be impossible to evaluate, for each scenario we provide an upper-bound by subtracting the dual solution and a lower-bound. We obtain a very close lowerbound on TAR for both TPC/TIC and TPC/IC combinations using the methodology developed in [50]. For brevity, we only describe the procedure for the case of TPC/TIC combination, and the same can be applied for the IC problem. Consider the problem $\mathcal{O}^{T I C}$. Applying some mathematical manipulation, 


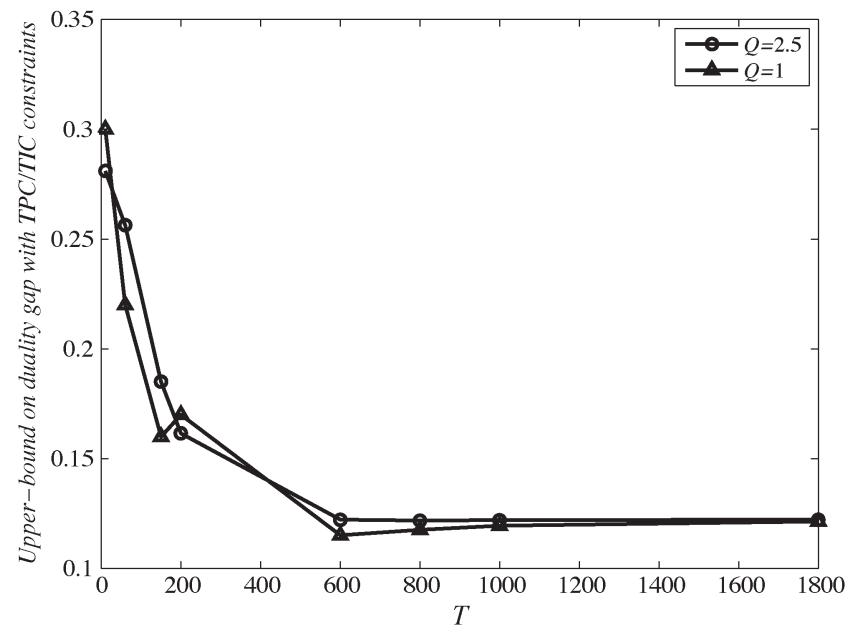

Fig. 2. Upper-bound on the duality gap with TPC/TIC constraints vs. T, for $P_{T}=10 \mathrm{~W}, M=2, K=3, N=16$.

$\mathcal{O}^{T I C}$ can be transformed to the following optimization problem assuming $\omega_{m}=1, \forall m$ :

Problem $\hat{\mathcal{O}}^{T I C}$ :

$$
\begin{aligned}
\frac{1}{2 T} \min _{\{\boldsymbol{p}[t]\}} \prod_{t, m, i} \frac{\sum_{\hat{m} \neq m} g_{m, i}[t] p_{\hat{m}, i}[t]+\bar{I}_{i, s^{m} \mid p}[t]+\sigma_{m}^{2}}{\sum_{\hat{m}=1}^{M} g_{m, i}[t] p_{\hat{m}, i}[t]+\bar{I}_{i, s^{m} \mid p}[t]+\sigma_{m}^{2}}, \\
\text { s.t. } \quad(T P C),(T I C) .
\end{aligned}
$$

We now obtain a solution of this optimization problem by utilizing Geometric Programming (GP) [32]. We first use a tight lower-bound on the objective function of $\hat{\mathcal{O}}^{T I C}$ based on the arithmetic-geometric mean (AGM) inequality as: $\sum_{\hat{m}} \kappa_{\hat{m}, i}[t] \alpha_{\hat{m}, i}[t] \geq \prod_{\hat{m}}\left(\alpha_{\hat{m}, i}[t]\right)^{\kappa_{\hat{m}, i}[t]}$, in which

$$
\alpha_{\hat{m}, i}[t]= \begin{cases}g_{m, i}[t] p_{\hat{m}, i}[t], & \hat{m} \neq m, \\ g_{m, i}[t] p_{m, i}[t]+\bar{I}_{i, s^{m} \mid p}[t]+\sigma_{m}^{2}, & \hat{m}=m,\end{cases}
$$

and $\quad \boldsymbol{\kappa}_{i}[t]=\left[\kappa_{1, i}[t], \kappa_{2, i}[t], \cdots, \kappa_{M, i}[t]\right] \quad$ and $\quad \mathbf{1}^{\prime} \boldsymbol{\kappa}_{i}[t]=1$. Therefore, $\varpi_{m, i}[t]=\sum_{\hat{m}} \alpha_{\hat{m}, i}[t]$ can be approximated by its

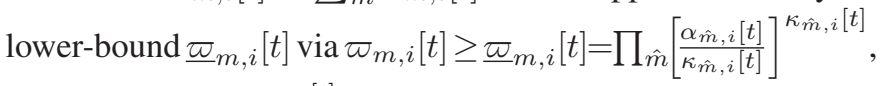
where $\kappa_{\hat{m}, i}[t]=\frac{\alpha_{\hat{m}, i}[t]}{\varpi_{\hat{m}, i}[t]}$. We now rewrite the lower-bound of the objective function as

$$
\prod_{t=1}^{T} \prod_{m=1}^{M} \prod_{i=1}^{N} \frac{\sum_{\hat{m} \neq m} g_{m, i}[t] p_{\hat{m}, i}[t]+\bar{I}_{i, s^{m} \mid p}[t]+\sigma_{m}^{2}}{\underline{\varpi}_{m, i}[t]} .
$$

Since the dominator is a posynomial function (product of posynomial functions is also a posynomial function), (36) is a posynomial function. Thus, based on this approximation, $\hat{\mathcal{O}}^{T I C}$ is transformed to a standard GP problem and can be solved by CVX [32].

Figs. 2 and 3 show the impact of length of time window, $T$, on the duality gap with TPC/TIC and TPC/IC combination constraints, respectively. Here, we fix the total number of available subcarriers at 16 . Fig. 2 shows that for the case of $1 \leq T \leq 600$, the duality gap reduces by increasing $T$, which is in line with our results. Note that for large enough $T$ this

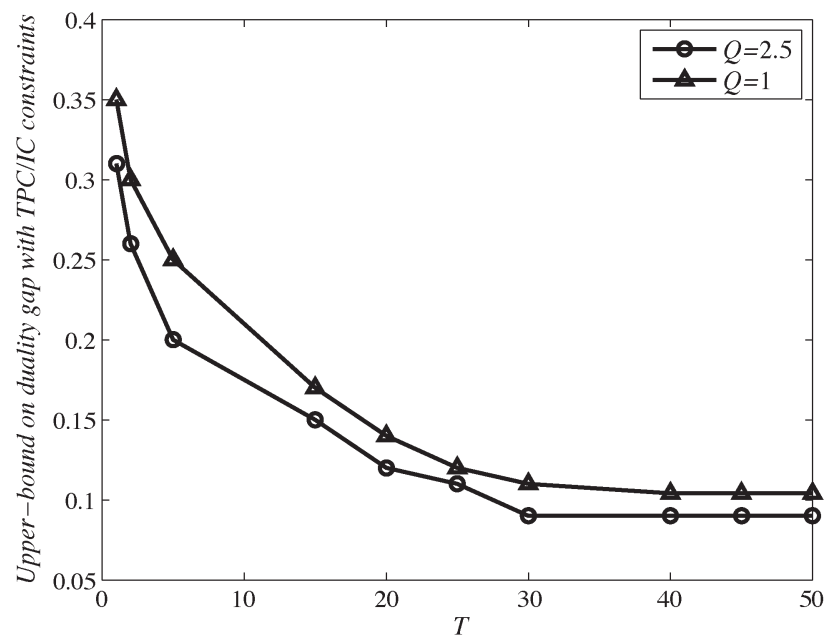

Fig. 3. Upper-bound on the duality gap with TPC/IC constraints $v s$. T, for $P_{T}=10 \mathrm{~W}, M=2, K=3, N=16$.

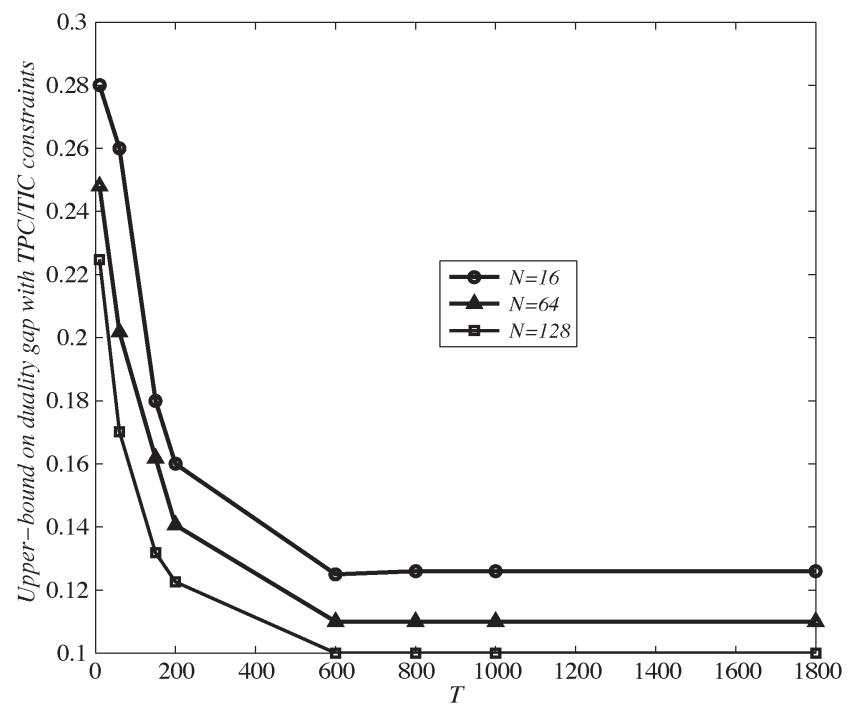

Fig. 4. Upper-bound on the duality gap with TPC/TIC constraints vs. T, for $P_{T}=10 \mathrm{~W}, Q_{m}^{L T}=2.5 \forall m, M=2, K=3$.

figure shows a nonzero duality gap due mainly to the inaccuracy of the thus-obtained optimal solution for $\hat{\mathcal{O}}^{T I C}$. We repeat the above for the case when the RRA includes TPC/IC combination constraints and plotted the results in Fig. 3. Again, increasing $T$ is found to reduce the duality gap. However, compared to Fig. 2, the reduction rate is much faster, as our analysis predicted.

What Fig. 4 illustrates is the behavior of the thus-provided upper-bound on the duality gap with TPC/TIC combination versus $T$ for different values of $N$. Increasing $N$ is also observed to reduce the duality gap, as predicted in [33]. The rate of decrement versus $T$, on the other hand, indicates that increasing $N$ is not as effective as increasing $T$. Note that in our simulation we set the coherence bandwidth such that the total available bandwidth is divided into $N=16$ flat fading sub-channels. Thus, increasing $N$ beyond 16 produces highly correlated subcarriers on each sub-channel.

Fig. 5 also represents the impact of the number of subcarriers $N$ on the duality gap with TPC/IC combination. Increasing $N$ inclines the duality gap, but the decrement rate is slower than that of time window $T$. 


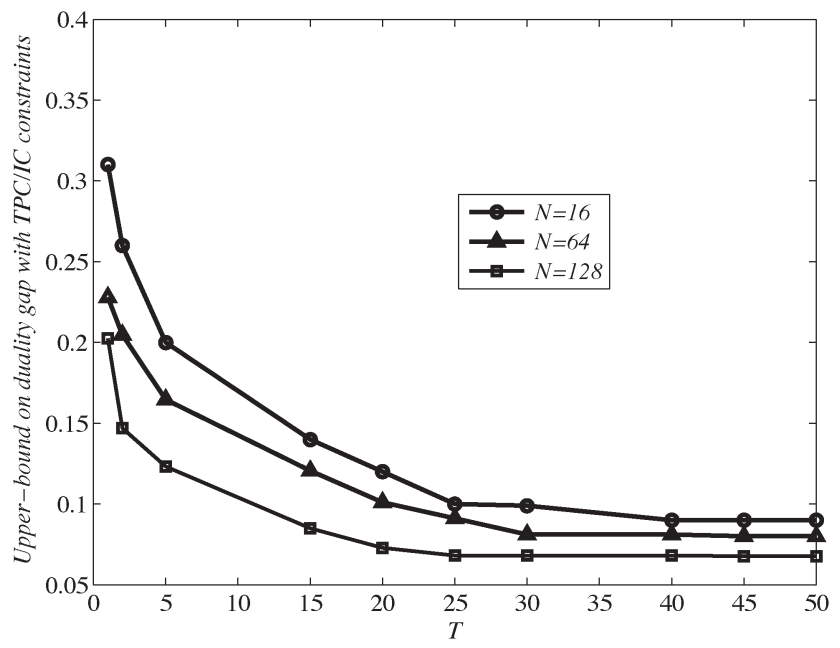

Fig. 5. Upper-bound on the duality gap with TPC/IC constraints $v s$. $T$, for $P_{T}=10 \mathrm{~W}, Q_{m}^{L T}=2.5 \forall m, M=2, K=3$.

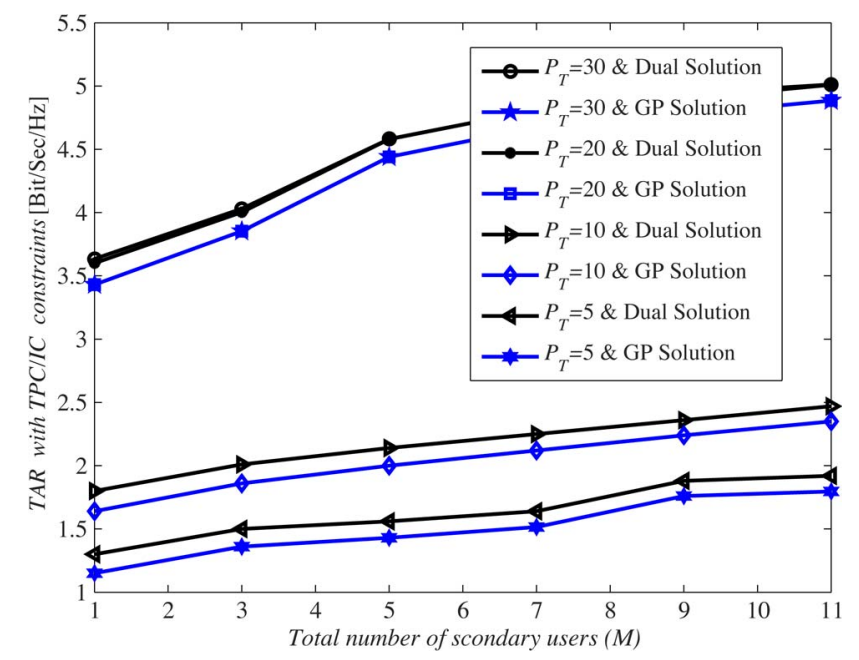

Fig. 6. Secondary service TAR with TPC/IC constraints $v s$. the number of the secondary users for $Q_{m}^{S T}=1 \mathrm{~W}, \forall m, K=7, T=20$.

\section{B. Performance of RRA}

We now study the secondary service TAR for the TPC/IC combination. The simulation results are plotted in Fig. 6. Our objective here is to understand the impact of the number of the secondary users as well as the secondary BS maximum average power on TAR. We set $Q_{m}^{S T}=1 \forall m$ and $T=50$. As shown in Fig. 6, by increasing $M$, we found an increase in TAR. As the number of the secondary users grows, the secondary BS allocates a larger number of the available subcarriers, thus increasing TAR. This is in line with what referred as multi-user diversity in multi-user cellular systems. Further, increasing $P_{T}$ generally enhances TAR. However, for large enough $P_{T}$, e.g., $P_{T}>20 \mathrm{~W}$ in this example, increasing $P_{T}$ does not change TAR due to the interference threshold constraint. In such cases, interference threshold is the dominant constraint. Fig. 6 further illustrates the comparison between the dual solution and the solution based on GP algorithm developed in (35). The main conclusion is that the difference between the two solutions are generally ignorable due to small duality gap predicted in Fig. 3 for $T>20$.

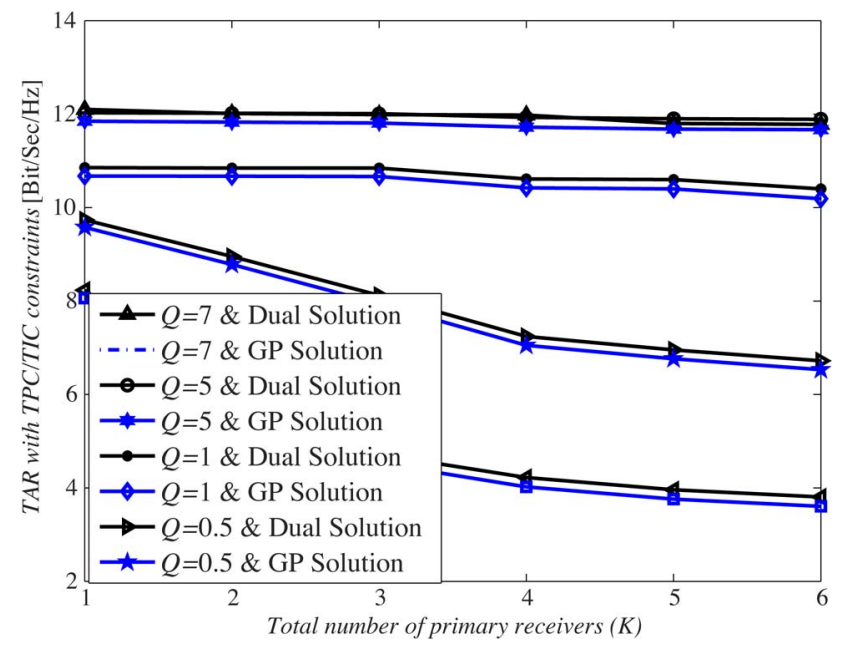

Fig. 7. Secondary service TAR with TPC/TIC constraints vs. the number of the primary receivers for $P_{T}=20 \mathrm{~W}, M=10, T=200$.

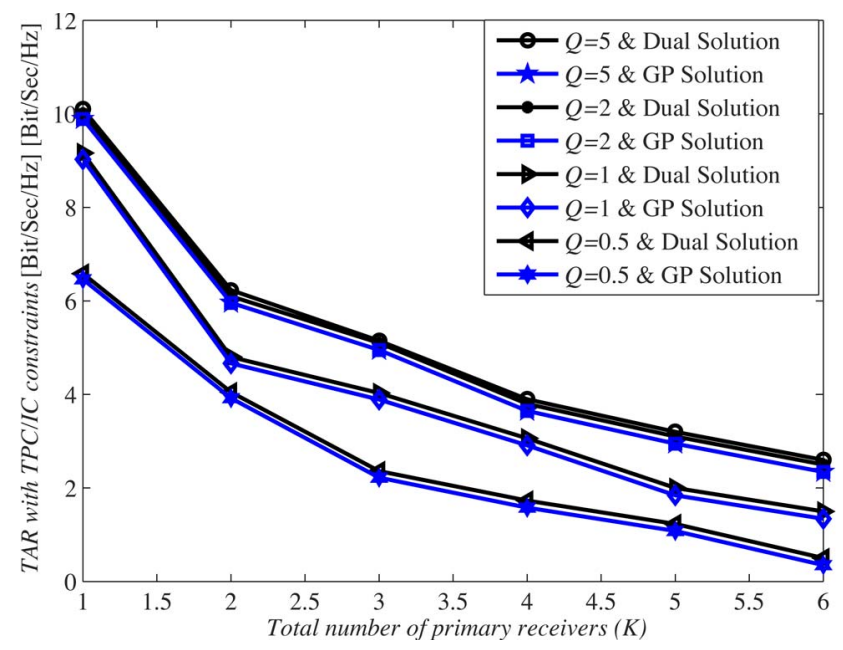

Fig. 8. Secondary service TAR with TPC/IC constraints $v s$. the number of the primary receivers for $P_{T}=20 \mathrm{~W}, M=10, T=30$.

Fig. 7 shows the impact of the number of the primary service receivers as well as interference threshold on the TAR with the TPC/TIC combination. By increasing TIC, TAR is also increased. However, for a large enough TIC (e.g., $Q=$ $Q_{k}^{L T} \geq 5$ ), increasing TIC does not increase TAR because TPC is dominant. Moreover, by increasing $K$, a decrease in TAR is observed when TIC is low enough. Since new TICs have to be satisfied, thus limiting further the allocated power at the secondary service, TAR is reduced by increasing $K$. The decreasing rate for a large enough TIC is almost negligible since in this case TPC is the dominant constraint. Thus, increasing the number of primary receivers does not affect TAR. Note that again, the difference between the dual solution and GP solution (35) is very small since for $T>200$ according to Fig. 2, the duality gap is very small.

Fig. 8 illustrates the impact of the number of primary service receivers as well as the IC on the TAR of secondary service with the TPC/IC combination. By increasing IC, TAR increases. However, for a large enough IC, i.e., $Q=Q_{k}^{S T} \geq 5$, increasing IC does not change TAR because TPC is the dominant constraint. Besides, by increasing $K$, there will be a decrease in 

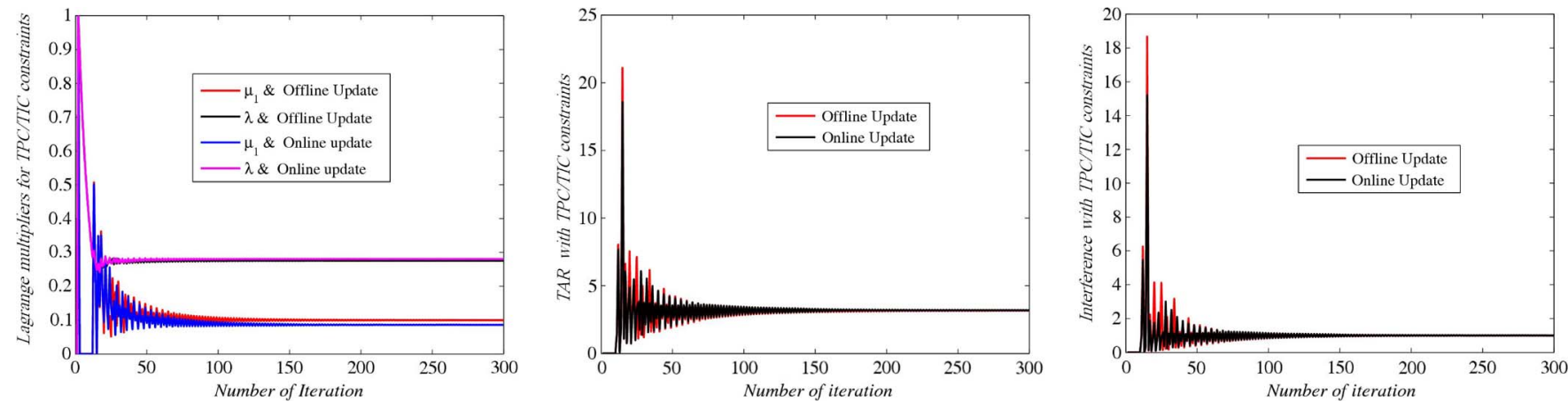

Fig. 9. Lagrangian multipliers for TPC/TIC constraints versus iteration count in off-line and online updates, where $P_{T}=5 \mathrm{~W}, M=4$, and $Q^{L T}=1$. (left) The y-axis shows values of two different Lagrangian multipliers $\lambda$ and $\mu_{1}$ for both off-line and online updates. (middle) Sum rate of the secondary service TAR. (right) Imposed interference on the primary service.
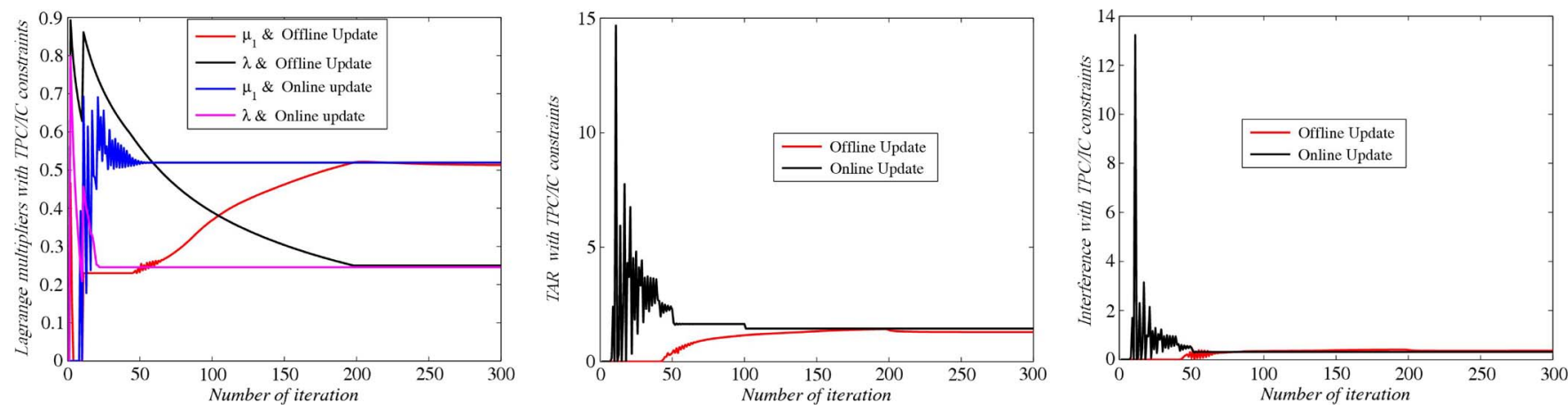

Fig. 10. Lagrangian multipliers for TPC/IC constraints versus iteration count in off-line and online updates, where $P_{T}=5 \mathrm{~W}, M=4$, and $Q^{S T}=1$. (left) The y-axis shows values of two different Lagrangian multipliers $\lambda$ and $\mu_{1}$ for both off-line and online updates. (middle) Sum rate of the secondary service TAR. (right) Imposed interference on the primary service.

TAR. By increasing $K$, IC constraints restrict the transmission power of the secondary service. However, note that in comparison with Fig. 7, even for large values of interference threshold the effect of increasing $K$ on TAR is negative. First, in the case of TPC/TIC combination in Fig. 8, the impact of interfering channel power gain is actually averaged out by summing up on time window $T$, and hence only $K$ different TICs have to be fulfilled in the optimization that does not limit the transmission power as in the case of TPC/IC combination. Second, for a large TIC, increasing $K$ does not reduce TAR since TPC is dominant. On the contrary, with TPC/IC combination, in aggregate $K T$, interference constraints have to be met. Increasing $K$ further adds up to the number of ICs, which dramatically limits the transmission power.

\section{Impact of Causality}

We also investigate the implementation of our resource allocation algorithms via online update of Lagrangian multipliers in Fig. 9 for the TPC/TIC combination. The left-hand side shows Lagrangian multipliers as a function of the iteration count for off-line and online updates. As observed in both online and off-line updates, the Lagrangian multipliers converge to the optimal values very quickly. This indicates the possibility of the causal implementation of the proposed algorithm in Section III. We also plotted the obtained achievable sum rate in the middle. Both online and off-line updates have the same TAR performance. Thus, the online implementation does not degrade the secondary service performance. More importantly, as the right-hand-side figure shows, the imposed interference at the primary service is the same in both off-line and on-line updates after sufficient iterations. Note that in the case of online update, the primary service may receive an unacceptable level of interference at the start of the window, e.g., when the number of iterations is less that 50 in this example. But, the online update can eventually guarantee the average TIC constraint to be met.

For the TPC/IC combination, Fig. 10 illustrates the results of the online and off-line updates of the Lagrangian multipliers. Lagrangian multipliers as a function of the iteration count for off-line and online updates are shown in the left-hand-side figure. In the both online and off-line updates, the Lagrangian multipliers converge to the optimal values very quickly. The middle figure illustrates the obtained achievable sum rate. As it is seen for large enough iterations both online and off-line updates have the same TAR performance. Thus, the online implementation does not degrade the secondary service performance. Finally, the right-hand-side figure shows the imposed interference at the primary service is the same in both off-line and on-line updates after sufficient iterations.

\section{CONCLUSION}

In this paper, we have studied the downlink RRA problem in a spectrum-sharing environment by introducing the time averaging window $T$. Specifically, we have considered DSCDMA/OFDM systems but the results are extendable to other scenarios. To achieve optimal RRA, we have utilized the dual 
decomposition method. We have proved that the duality gap between the solution obtained by adopting the dual decomposition and the optimal solution vanishes at least by an exponential rate of $T$, which is the time scale of RRA operation. This result assures that for a large enough $T$, the dual decomposition method provides solutions very close to the optimal solution. We have also observed that the rate of vanishing the duality gap in TPC/IC is much higher than that in TPC/TIC. We have proved that OFDMA is asymptotically the optimal subcarrier assignment in resource allocation problems for both TPC/TIC and TPC/IC constraints. Under each of these two constraints, the optimal power allocation has also been derived. Extensive simulations have been conducted to study the impact of various system parameters, especially $T$, on the secondary service performance. Our simulation results have confirmed that the proposed RRA algorithms are practical and possible to run online without harming the convergence and optimality. These findings are of practical significance for two reasons. First, when the number of available sub-channels is limited, which is usually the case in spectrum-sharing systems, it is possible to reduce the duality gap associated with using OFDMA as much as possible by increasing the time window $T$. We have shown that OFDMA is the asymptotically optimal subcarrier assignment. Second, the resource-allocation algorithms developed in this paper are robust in practical situations, particularly when the transmitter has only on-the-fly CSI knowledge. Our simulation results have confirmed that the Lagrangian multipliers converge quickly to the theoretical values.

\section{APPENDIX A}

\section{PROOF OF THEOREM 1}

In its general form, the convex feasible power set, $\mathcal{P}[t]$, is not in closed-form and not bounded, so it is not a compact set. Therefore, there is no upper-bound of the duality gap. In what follows, we show that considering the actual system results in a compact $\mathcal{P}[t]$, where an upper-bound can be obtained.

Assume that the transmission power of each subcarrier is limited by imposing a large enough positive cutoff power denoted by $p_{\max }$. We then define a new power set, $\tilde{\mathcal{P}}[t] \triangleq \tilde{\mathcal{P}}_{1}[t] \times \ldots \times$ $\tilde{\mathcal{P}}_{N}[t]$, where $\tilde{\mathcal{P}}_{i}[t]=\left\{\boldsymbol{p}_{i}[t] \mid 0 \leq p_{m, i}[t] \leq p_{\max }, \forall m\right\}$. If we consider a new optimization problem by substituting $\tilde{\mathcal{P}}[t]$ with $\mathcal{P}[t]$ and considering a very high value for $p_{\max }$, the solutions of the new problem are the same as $\mathcal{O}^{T I C}$. For $\mathcal{O}^{T I C}$ existence of at least one feasible solution is guaranteed for both power sets $\tilde{\mathcal{P}}[t]$ and $\mathcal{P}[t]$. For instance, OFDMA subcarrier assignment can be considered as a feasible solution. Therefore, since TPC and TIC are continuous and convex, and due to the fact that feasible power set is also convex, the assumptions A1(A3 of Proposition 5.26 in [51] are satisfied (see Appendix B). Consequently, the following holds:

$$
\inf (D(\lambda, \boldsymbol{\mu}))-\sup \left(\mathcal{O}^{T I C}\right) \leq \frac{\delta}{T}
$$

for a bounded and positive $\delta$. Equation (37) indicates that for $T \rightarrow \infty$, the duality gap between the solutions of the primal and dual problems approaches 0 . Based on the result of
Proposition 5.26 in [51] (see Appendix B), we know that parameter $\delta$ in (37) is equal to $(K+2) E$, where $K$ is the number of primary receivers, $E=\max _{t=1, \ldots, T}\left\{\rho_{t}\right\}$ and $\rho_{t}$ at each time instant $t$ is also upper-bounded as

$$
\rho_{t} \leq \sup \{r[t] \mid \tilde{\mathcal{P}}[t]\}-\inf \{r[t] \mid \tilde{\mathcal{P}}[t]\}
$$

where $r[t]=\sum_{m=1}^{M} \omega_{m} \sum_{i=1}^{N} \frac{1}{2} \log \left(1+\gamma_{m, i}[t]\right)$. It is easy to observe that $\inf \{r[t] \mid \tilde{\mathcal{P}}[t]\}=0$. Noting (37), an upper-bound for $\mathcal{D}(\varepsilon)$ can be suggested as

$$
\begin{aligned}
\mathcal{D}(\varepsilon) & \leq \mathbb{P}\left\{\max _{t=1, \ldots, T}\{\sup \{r[t] \mid \tilde{\mathcal{P}}[t]\}\}>\frac{T \varepsilon}{K+2}\right\} \\
& =1-\prod_{t=1}^{T}\left(\mathbb{P}\left\{\sup \{r[t] \mid \tilde{\mathcal{P}}[t]\} \leq \frac{T \varepsilon}{K+2}\right\}\right) \\
& =1-\left[\mathbb{P}\left\{\sup \{r \mid \tilde{\mathcal{P}}\} \leq \frac{T \varepsilon}{K+2}\right\}\right]^{T} .
\end{aligned}
$$

where $\sup \{r \mid \tilde{\mathcal{P}}\}$ is $\max _{\tilde{\mathcal{P}}} \sum_{m=1}^{M} \omega_{m} \sum_{i=1}^{N} \frac{1}{2} \log \left(1+\gamma_{m, i}\right)$. We have

$$
\begin{aligned}
\max _{\tilde{\mathcal{P}}} & \sum_{m, i} \frac{\omega_{m}}{2} \log \left(1+\gamma_{m, i}\right) \\
& \leq \sum_{m, i} \frac{\omega_{m}}{2} \log \left(1+\frac{p_{\max } g_{m, i}}{\bar{I}_{i, s^{m} \mid p}+\sigma_{m}^{2}}\right) \\
& \leq \sum_{m, i} \frac{\omega_{m}}{2} \log \left(1+\frac{p_{\max } g_{m, i}}{\bar{I}_{i, s^{m} \mid p}}\right) .
\end{aligned}
$$

Therefore,

$$
\sup \{r \mid \tilde{\mathcal{P}}\} \leq \sum_{i=1}^{N} \sum_{m=1}^{M} \frac{\omega_{m}}{2} \log \left(1+\frac{1}{\frac{\bar{I}_{i, s}{ }^{m}}{p_{\max } g_{m, i}}}\right) .
$$

To obtain the upper-bound, we note in (38),

$$
\begin{aligned}
\mathbb{P} & \left\{\sup \{r \mid \tilde{\mathcal{P}}\}>\frac{T \varepsilon}{K+2}\right\} \\
& \leq \mathbb{P}\left\{\sum_{m=1}^{M} \sum_{i=1}^{N} \log \left(1+\frac{p_{\max }}{\frac{\bar{I}_{i, s} \mid p}{g_{m, i}}}\right)>\frac{T \varepsilon}{K+2}\right\} \\
& \leq \mathbb{P}\left\{N M \log \left(1+p_{\max } \max _{m} \max _{i} \frac{g_{m, i}}{\bar{I}_{i, s^{m} \mid p}}\right)>\frac{T \varepsilon}{K+2}\right\} \\
& =\mathbb{P}\left\{\max _{m=1, \ldots, M} \max _{i=1, \ldots, N} \frac{g_{m, i}}{\bar{I}_{i, s^{m}} \mid p}>\frac{e^{\frac{T_{\varepsilon}}{N M(K+2)}}-1}{p_{\max }}\right\} \\
& =1-\mathbb{P}\left\{\max _{m=1, \ldots, M} \max _{i=1, \ldots, N} \frac{g_{m, i}}{\bar{I}_{i, s^{m}} \mid p} \leq \frac{e^{\frac{T \varepsilon}{N M(K+2)}}-1}{p_{\max }}\right\} \\
& =1-\prod_{m=1}^{M} \prod_{i=1}^{N} \mathbb{P}\left\{\frac{g_{m, i}}{\bar{I}_{i, s^{m}} \mid p} \leq \frac{e^{\frac{T \varepsilon}{N M(K+2)}}-1}{p_{\max }}\right\} \\
& =1-\prod_{m=1}^{M} \prod_{i=1}^{N}\left(1-\mathbb{P}\left\{\frac{g_{m, i}}{\bar{I}_{i, s^{m}} \mid p}>\frac{e^{\frac{T \varepsilon}{N M(K+2)}}-1}{p_{\max }}\right\}\right) .
\end{aligned}
$$


The channel power gain $g_{m, i}$ is exponentially distributed with mean value of $1 / \mu_{m, i}$. Therefore,

$$
\begin{aligned}
& \mathbb{P}\left\{\sup \{r \mid \tilde{\mathcal{P}}\}>\frac{T \varepsilon}{K+2}\right\} \\
& \quad \leq 1-\prod_{m=1}^{M} \prod_{i=1}^{N}\left(1-e^{-\frac{e^{\frac{T \varepsilon}{N M(K+2)}}-1}{p_{\max }} \mu_{m, i} \bar{I}_{i, s}{ }^{m} \mid p}\right) \\
& \quad \leq 1-\left(1-e^{-\frac{e^{\frac{T \varepsilon}{N M(K+2)}}-1}{p_{\max }} \min _{m, i}\left\{\mu_{m, i} \bar{I}_{i, s}{ }^{\prime} \mid p\right.}\right)^{M N} .
\end{aligned}
$$

Using (41) in (38) yields,

$$
\mathcal{D}(\varepsilon) \leq 1-\left(1-e^{-\frac{e^{\frac{T \varepsilon}{N M(K+2)}}-1}{p_{\max }} \min _{m, i}\left\{\mu_{m, i} \bar{I}_{i, s} \mid p\right\}}\right)^{M N T} .
$$

We then show that there exists a $T(\xi)$, where for $T>T(\xi)$, $\mathbb{P}\left\{\sup \{r \mid \tilde{\mathcal{P}}\}>\frac{T \varepsilon}{K+2}\right\} \leq \xi$, thus $\mathcal{D}(\varepsilon) \leq 1-(1-\xi)^{T} . T(\xi)$ is then obtained from the bound in (41) as:

$$
e^{-\frac{e^{\frac{T \varepsilon}{N M(K+2)}}-1}{p_{\max }} \min _{m, i}\left\{\mu_{m, i} \bar{I}_{i, s}{ }_{\mid p}\right\}} \leq 1-(1-\xi)^{\frac{1}{M N}},
$$

or equivalently

$$
T \geq \frac{N M(K+2)}{\varepsilon} \ln \left(\frac{1+p_{\max } \ln \frac{1}{1-(1-\xi) \frac{1}{M N}}}{\min _{m, i}\left\{\mu_{m, i} \bar{I}_{i, s^{m}} \mid p\right\}}\right)=T(\xi) .
$$

This implies that if $T \rightarrow \infty, \frac{e^{\frac{T \varepsilon}{N M(K+2)}}-1}{p_{\max }} \gg 1$ holds, and thus

$$
1-e^{-\frac{e^{\frac{T \varepsilon}{N M(K+2)}}-1}{p_{\max }} \min _{m, i}\left\{\mu_{m, i} \bar{I}_{i, s^{m}} \mid p\right\}} \rightarrow 1 .
$$

Finally,

$$
\begin{aligned}
1- & e^{-\frac{e^{\frac{T \varepsilon}{N M(K+2)}}-1}{p_{\max }} \min _{m, i}\left\{\left.\mu_{m, i} \bar{I}_{i, s}\right|_{p}\right\}} \\
& \geq 1-e^{-\left(\frac{T \varepsilon}{N M(K+2) p_{\max }}+\frac{T^{2} \varepsilon^{2}}{N^{2} M^{2}(K+2)^{2} p_{\max }}\right) \min _{m, i}\left\{\mu_{m, i} \bar{I}_{i, s}{ }_{\mid p}\right\}} \\
& \geq 1-e^{-\left(\frac{\varepsilon}{N M(K+2)}+\frac{T \varepsilon^{2}}{N^{2} M^{2}(K+2)^{2}}\right) \min _{m, i}\left\{\left.\mu_{m, i} \bar{I}_{i, s}\right|_{\mid p}\right\}} \\
& \geq 1-e^{-T \frac{\varepsilon^{2} \min _{m, i}\left\{\left.\mu_{m, i} \bar{I}_{i, s}\right|_{\mid p}\right\}}{N^{2} M^{2}(K+2)^{2}}} .
\end{aligned}
$$

Let $c=\frac{\min _{m, i}\left\{\mu_{m, i} \bar{I}_{i, s} m_{\mid p}\right\}}{N^{2} M^{2}(K+2)^{2}}$, it is then confirmed that $\mathcal{D}(\varepsilon) \leq$ $1-\left(1-e^{-c \varepsilon^{2} T}\right)^{M N T}$, which implies the decrement rate is $\left(1-e^{-c \varepsilon^{2} T}\right)^{M N T}$.

\section{APPENDiX B}

Consider the following generic optimization problem

Problem $\mathcal{P}: \min _{\left\{\boldsymbol{x}_{t}\right\} \in\left\{\mathcal{X}_{t}\right\}} \sum_{t=1}^{T} f_{t}\left(\boldsymbol{x}_{t}\right), \quad$ s.t. $\quad \sum_{t=1}^{T} \boldsymbol{h}_{t}\left(\boldsymbol{x}_{t}\right) \leq \boldsymbol{b}$,

where $T$ is a positive integer, $\boldsymbol{b} \in \mathbb{R}^{m}$ for given positive integer $m, \mathcal{X}_{t} \in \mathbb{R}^{q_{t}}$ for given positive integers $q_{t}$. Denoting $\operatorname{conv}\left(\mathcal{X}_{t}\right)$ as the convex hull of $\boldsymbol{\mathcal { X }}_{t}, f_{t}\left(\boldsymbol{x}_{t}\right)$ and $\boldsymbol{h}_{t}\left(\boldsymbol{x}_{t}\right)$ are functions defined as $f_{t}\left(\boldsymbol{x}_{t}\right): \operatorname{conv}\left(\boldsymbol{\mathcal { X }}_{t}\right) \rightarrow \mathbb{R}$ and $\boldsymbol{h}_{t}\left(\boldsymbol{x}_{t}\right): \operatorname{conv}\left(\boldsymbol{\mathcal { X }}_{t}\right) \rightarrow$ $\mathbb{R}^{m}$, where $\operatorname{conv}(\boldsymbol{x})$ is the convex haul of set $\boldsymbol{x}$. Now, consider the following three assumptions: A1: There is at least one feasible solution to $\mathcal{P}$; $\mathbf{A 2}$ : For each $t$, the subset of $\mathbb{R}^{q_{t}+l+1}$, $\left\{\left(\boldsymbol{x}_{t}, \boldsymbol{h}_{t}\left(\boldsymbol{x}_{t}\right), f_{t}\left(\boldsymbol{x}_{t}\right)\right) \mid \boldsymbol{x}_{t} \in \mathcal{X}_{t}\right\}$, is compact; A3: For each $t$, given any vector $\boldsymbol{y} \in \operatorname{conv}\left(\mathcal{X}_{t}\right)$ there exists $x \in \mathcal{X}_{t}$ such that $\boldsymbol{h}_{t}\left(\boldsymbol{x}_{t}\right) \preceq \tilde{\boldsymbol{h}}_{t}\left(\boldsymbol{y}_{t}\right)$, where $\tilde{\boldsymbol{h}}_{t}\left(\boldsymbol{x}_{t}\right): \operatorname{conv}\left(\boldsymbol{\mathcal { X }}_{t}\right) \rightarrow \mathbb{R}^{m}$. Note that A2 is satisfied if $\boldsymbol{X}_{t}$ is a compact set and both $f_{t}\left(\boldsymbol{x}_{t}\right)$ and $\boldsymbol{h}_{t}\left(\boldsymbol{x}_{t}\right)$ are continuous functions on $\boldsymbol{X}_{t}$. $\mathbf{A 3}$ is guaranteed whenever $\mathcal{X}_{t}$ is convex and each component of function $\boldsymbol{h}_{t}\left(\boldsymbol{x}_{t}\right)$ is convex.

Now, define the dual problem corresponding to $\mathcal{P}$ as

Problem D :

$$
\max _{\boldsymbol{\nu} \succeq 0} D(\boldsymbol{\nu})=\inf _{\left\{\boldsymbol{x}_{t}\right\} \in\left\{\mathcal{X}_{t}\right\}} \sum_{t=1}^{T}\left[f_{t}\left(\boldsymbol{x}_{t}\right)+\boldsymbol{\nu}^{H} \boldsymbol{h}_{t}\left(\boldsymbol{x}_{t}\right)\right]-\boldsymbol{\nu}^{H} \boldsymbol{b} .
$$

Proposition 2 [Proposition 5.26 in [51]]: Under assumptions A1-A3, $\inf (P)-\sup (D) \leq(l+1) E$ holds, where $E=\max _{t=1, \ldots, T} \rho_{t}$, and $\rho_{t} \leq \sup \left(f_{t}\left(\boldsymbol{x}_{t}\right)\right)-\inf \left(f_{t}\left(\boldsymbol{x}_{t}\right)\right)$.

Corollary 2: If $E$ and $l$ are not functions of $t$, Proposition 1 implies that the optimization problem

Problem P 1 : $\min _{\left\{\boldsymbol{x}_{t}\right\} \in\left\{\mathcal{X}_{t}\right\}} \frac{1}{T} \sum_{t=1}^{T} f_{t}\left(\boldsymbol{x}_{t}\right)$, s.t. $\sum_{t=1}^{T} \boldsymbol{h}_{t}\left(\boldsymbol{x}_{t}\right) \leq \boldsymbol{b}$,

has a duality gap estimate of $\inf (P 1)-\sup (D 1) \leq \frac{(l+1) E}{T}$, which approaches 0 by $T \rightarrow \infty$.

\section{APPENDIX C \\ PROOF OF THEOREM 2}

Here we also utilize the dual decomposition method and introduce set $\mathcal{Q}[t]$, where for given time instant $t$, it comprises the intersection between the feasible power allocation set, $\mathcal{P}[t]$, and the set corresponding to the IC constraint. Problem $\mathcal{O}^{I C}$ is then reduced to

$$
\begin{array}{r}
R=\max _{\{\boldsymbol{p}[t]\} \in\{\mathcal{Q}[t]\}} \frac{1}{T} \sum_{t=1}^{T} \sum_{m=1}^{M} \omega_{m} \sum_{i=1}^{N} \frac{1}{2} \log \left(1+\gamma_{m, i}[t]\right), \\
\text { s.t. } \quad \frac{1}{T} \sum_{t=1}^{T} \sum_{m=1}^{M} \sum_{i=1}^{N} p_{m, i}[t] \leq P_{T} .
\end{array}
$$

According to the IC constraint, $\forall t$ the following inequality holds:

$$
\begin{aligned}
& \sum_{i=1}^{N} h_{i}^{k}[t] \sum_{m=1}^{M} p_{m, i}[t] \leq \sum_{i=1}^{N} \max _{t} h_{i}^{k}[t] \sum_{m=1}^{M} p_{m, i}[t] \\
& \leq N \max _{i} \max _{t} h_{i}^{k}[t] \sum_{m=1}^{M} p_{m, i}[t] \leq Q_{k}^{S T}, \quad \forall k .
\end{aligned}
$$

Consequently,

$$
p_{m, i}[t] \leq \frac{\max _{k} Q_{k}^{S T}}{N \max _{i, k} \max _{t} h_{i}^{k}[t]} .
$$

Therefore, $\forall t, \mathcal{Q}[t]$, is a compact set. Thus, we have $\inf (D(\lambda))-\sup \left(\mathcal{O}^{I C}\right) \leq \frac{\alpha}{T}$, (see the Appendix B) where $\lambda$ 
is the Lagrangian multiplier corresponding to the TPC constraint, $\alpha$ is a finite positive number, and $D(\lambda)$ is the dual function. In what follows, we derive an upper-bound for $\mathcal{D}(\varepsilon)$. Based on the result of Proposition 5.26 in [51], we know that parameter $\alpha$ in (37) is equal to $(K+2) E$ where $E$ is $E=$ $\max _{t=1, \ldots, T}\left\{\rho_{t}\right\}$, and $\rho_{t}$ at each time instant $t$ is upper-bounded as $\rho_{t} \leq \sup \{r[t] \mid \mathcal{Q}[t]\}-\inf \{r[t] \mid \mathcal{Q}[t]\}$. It is easy to see that $\inf \{r[t] \mid \mathcal{Q}[t]\}=0$, so

$$
\mathcal{D}(\varepsilon) \leq 1-\left[\mathbb{P}\left\{\sup \{r \mid \mathcal{Q}\} \leq \frac{T \varepsilon}{K+2}\right\}\right]^{T} .
$$

By considering the definition of $r$, and dropping the time index $t$ for brevity, $\sup \{r \mid \mathcal{Q}\}$ is

$$
\max _{p \in \mathcal{Q}} \sum_{m=1}^{M} \omega_{m} \sum_{i=1}^{N} \frac{1}{2} \log \left(1+\gamma_{m, i}\right) .
$$

Instead of solving the above optimization problem, we propose an upper-bound for the solution. Note that

$$
\sum_{i=1}^{N} h_{i}^{k} \sum_{m=1}^{M} p_{m, i} \leq N \max _{i} h_{i}^{k} \sum_{m=1}^{M} p_{m, i} \leq Q_{k}^{S T}, \forall k .
$$

Furthermore, it can be seen that

$$
\sum_{m=1}^{M} p_{m, i} \leq \max _{k} \frac{Q_{k}^{S T}}{N \max _{i} h_{i}^{k}} \triangleq p_{\max } .
$$

Consequently, to obtain an upper-bound of $\sup \{r \mid \mathcal{Q}\}$, we require to solve the following optimization problem

$$
\max _{p_{m, i} \leq p_{\max }, \forall m, i} \sum_{m=1}^{M} \omega_{m} \sum_{i=1}^{N} \frac{1}{2} \log \left(1+\gamma_{m, i}\right) .
$$

From the above optimization problem, we can conclude

$$
\begin{aligned}
& \max _{p_{m, i} \leq p_{\max }, \forall m, i} \sum_{m, i} \frac{\omega_{m}}{2} \log \left(1+\gamma_{m, i}\right) \\
\leq & \sum_{m, i} \frac{\omega_{m}}{2} \log \left(1+\frac{p_{\max } g_{m, i}}{\bar{I}_{i, s^{m} \mid p}+\sigma_{m}^{2}}\right) \\
\leq & \sum_{m, i} \frac{\omega_{m}}{2} \log \left(1+\frac{p_{\max } g_{m, i}}{\bar{I}_{i, s^{m} \mid p}}\right) .
\end{aligned}
$$

Therefore,

$\sup \{r \mid \mathcal{Q}\} \leq \sum_{i=1}^{N} \sum_{m=1}^{M} \frac{\omega_{m}}{2} \log \left(1+\frac{\max _{k} \frac{Q_{k}^{S T}}{N \max _{j} h_{j}^{k}}}{\frac{\bar{I}_{i, s}{ }^{m} \mid p}{g_{m, i}}}\right)$.

We utilize (48) to derive an upper-bound for $\mathbb{P}\left\{\sup \{r \mid \mathcal{Q}\}>\frac{T \varepsilon}{K+2}\right\}$ as:

$\mathbb{P}\left\{\sup \{r \mid \mathcal{Q}\}>\frac{T \varepsilon}{K+2}\right\}$

$\leq \mathbb{P}\left\{\sum_{m=1}^{M} \sum_{i=1}^{N} \log \left(1+\frac{\max _{k} \frac{Q_{k}^{S T}}{N \max _{j} h_{j}^{k}}}{\frac{\left.\bar{I}_{i, s}\right|_{\mid p}}{g_{m, i}}}\right)>\frac{T \varepsilon}{K+2}\right\}$

$$
\begin{aligned}
& \leq \mathbb{P}\left\{M N \log \left(1+\max _{m} \max _{i} \frac{\max _{k} \frac{Q_{k}^{S T}}{N \max _{j} h_{j}^{k}}}{\frac{\bar{I}_{i, s} m \mid p}{g_{m, i}}}\right)>\frac{T \varepsilon}{K+2}\right\} \\
& =\mathbb{P}\left\{\max _{m} \max _{i} \frac{\max _{k} \frac{Q_{k}^{S T}}{N \max _{j} h_{j}^{k}}}{\frac{\bar{I}_{i, s^{m} \mid p}}{g_{m, i}}}>e^{\frac{T_{\varepsilon}}{M N(K+2)}}-1\right\} \\
& =1-\prod_{m=1}^{M} \prod_{i=1}^{N} \mathbb{E} \mathbb{P}\left\{\frac{g_{m, i}}{\bar{I}_{i, s^{m} \mid p}} \leq \frac{N\left(e^{\frac{T \varepsilon}{N M(K+2)}}-1\right)}{\max _{k} \min _{j} \frac{Q_{k}^{S T}}{h_{j}^{k}}} \mid h_{j}^{k}, \forall j, k\right\} .
\end{aligned}
$$

Here, $\mathbb{E}[x]$ is the expectation of random value $x$. Channel power gain $g_{m, i}$ has an exponential distribution with mean value of $1 / \mu_{m, i}$. Therefore,

$$
\begin{aligned}
& \mathbb{P}\left\{\sup \{r \mid \mathcal{Q}\}>\frac{T \varepsilon}{K+2}\right\} \\
& \leq 1-\prod_{m=1}^{M} \prod_{i=1}^{N} \mathbb{E}\left[1-e^{-\left.N \frac{e^{\frac{T \varepsilon}{N M(K+2)}}-1}{\max _{k} \min _{j} \frac{Q_{k}^{S T}}{h_{j}^{k}}} \mu_{m, i} \bar{I}_{i, s}\right|_{p}}\right] .
\end{aligned}
$$

Channel power gain $h_{i}^{k}$ for $\forall k, i$ are exponentially distributed random variables with corresponding mean value $1 / \mu_{k, i}$. Therefore, (49) is further reduced to

$$
\begin{aligned}
\mathbb{P}\left\{\sup \{r \mid \mathcal{Q}\}>\frac{T \varepsilon}{K+2}\right\} & \leq 1-\prod_{m=1}^{M} \prod_{i=1}^{N}\left[1-\sum_{j=1}^{N} \prod_{l \neq j}\left(\sum_{k=1}^{K} \mu_{k, j} Q_{k}^{S T}\right)\right. \\
& \times\left\{\int_{0}^{\infty} e^{-\left(\left.N\left(e^{\frac{T \varepsilon}{N M(K+2)}}-1\right) \mu_{m, i} \bar{I}_{i, s}\right|_{\mid p}+\sum_{k=1}^{K} \mu_{k, j} Q_{k}^{S T}\right) \omega} d \omega\right. \\
-\int_{0}^{\infty} e & -\left(e^{\left.N\left(\frac{T \varepsilon}{N M(K+2)}-1\right) \mu_{m, i} \bar{I}_{i, s} m \mid p+\sum_{k=1}^{K}\left(\mu_{k, j}+\mu_{k, l}\right) Q_{k}^{S T}\right) \omega} d \omega\right\} \\
=1 & -\prod_{m=1}^{M} \prod_{i=1}^{N}\left[1-\sum_{j=1}^{N} \prod_{l \neq j}\left(\sum_{k=1}^{K} \mu_{k, j} Q_{k}^{S T}\right)\right.
\end{aligned}
$$$$
\times\left[\frac{1}{N\left(e^{\frac{T \varepsilon}{N M(K+2)}}-1\right) \mu_{m, i} \bar{I}_{i, s^{m} \mid p}+\sum_{k=1}^{K} \mu_{k, j} Q_{k}^{S T}}\right.
$$$$
\left.\left.-\frac{1}{N\left(e^{\frac{T \varepsilon}{N M(K+2)}}-1\right) \mu_{m, i} \bar{I}_{i, s^{m} \mid p}+\sum_{k=1}^{K}\left(\mu_{k, j}+\mu_{k, l}\right) Q_{k}^{S T}}\right]\right]
$$ 


$$
\begin{aligned}
& \leq 1-\prod_{m=1}^{M} \prod_{i=1}^{N}\left[1-\sum_{j=1}^{N} \prod_{l \neq j} \frac{\sum_{k=1}^{K} \mu_{k, j} Q_{k}^{S T}}{\min _{m, i} \mu_{m, i} \bar{I}_{i, s^{m} \mid p}}\right. \\
& \times\left[\frac{1}{N\left(e^{\frac{T \varepsilon}{N M(K+2)}}-1\right)+\frac{\sum_{k=1}^{K} \mu_{k, j} Q_{k}^{S T}}{\left.\min _{m, i} \mu_{m, i} \bar{I}_{i, s}\right|_{p}}}\right.
\end{aligned}
$$

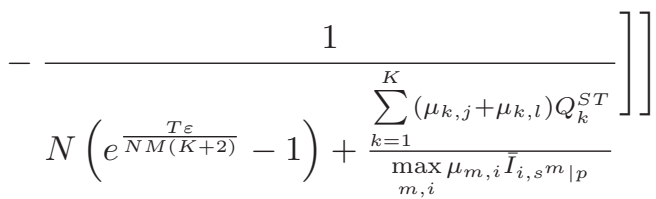

$$
\begin{aligned}
& =1-\left[1-\sum_{j=1}^{N} \prod_{l \neq j} \frac{\sum_{k=1}^{K} \mu_{k, j} Q_{k}^{S T}}{\min _{m, i} \mu_{m, i} \bar{I}_{i, s^{m} \mid p}}\right. \\
& \times\left[\frac{1}{N\left(e^{\frac{T \varepsilon}{N M(K+2)}}-1\right)+\frac{\sum_{k=1}^{K} \mu_{k, j} Q_{k}^{S T}}{\left.\min _{m, i} \mu_{m, i} I_{i, s}\right|_{p}}}\right. \\
& \left.\left.-\frac{1}{N\left(e^{\frac{T \varepsilon}{N M(K+2)}}-1\right)+\frac{\sum_{k=1}^{K}\left(\mu_{k, j}+\mu_{k, l}\right) Q_{k}^{S T}}{\max _{m, i} \mu_{m, i} \bar{I}_{i, s} m p}}\right]\right]^{M N} \\
& \leq 1-\left[1-\sum_{j=1}^{N} \prod_{l \neq j} \frac{\max _{j} \sum_{k=1}^{K} \mu_{k, j} Q_{k}^{S T}}{\min _{m, i} \mu_{m, i} \bar{I}_{i, s^{m} \mid p}}\right. \\
& \times\left[\frac{1}{N\left(e^{\frac{T \varepsilon}{N M(K+2)}}-1\right)+\frac{\min _{j} \sum_{k=1}^{K} \mu_{k, j} Q_{k}^{S T}}{\min _{m, i} \mu_{m, i} \bar{I}_{i, s}{ }^{m} \mid p}}\right.
\end{aligned}
$$

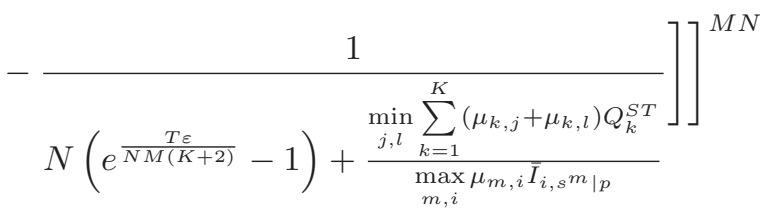

$$
\begin{aligned}
& \leq 1-\left[1-d\left[\frac{1}{e^{c^{\prime} \varepsilon T}+a}-\frac{1}{e^{c^{\prime} \varepsilon T}+b}\right]\right]^{M N^{2}}
\end{aligned}
$$

where $c^{\prime}=\frac{1}{N M(K+2)}$. Further, parameters $a, b$, and $d$ are respectively defined as

$$
\begin{gathered}
a=\frac{\min _{j} \sum_{k=1}^{K} \mu_{k, j} Q_{k}^{S T}}{N \min _{m, i} \mu_{m, i} \bar{I}_{i, s^{m} \mid p}}-1, \\
b=\frac{\min _{j, l} \sum_{k=1}^{K}\left(\mu_{k, j}+\mu_{k, l}\right) Q_{k}^{S T}}{N \max _{m, i} \mu_{m, i} \bar{I}_{i, s^{m} \mid p}}-1, \\
d=\sum_{j=1}^{N} \prod_{l \neq j} \frac{\sum_{k=1}^{K} \mu_{k, j} Q_{k}^{S T}}{\min _{m, i} \mu_{m, i} \bar{I}_{i, s^{m} \mid p}} .
\end{gathered}
$$

For $T \gg \tilde{T}$, where $\tilde{T}=\frac{N M(K+2)}{\varepsilon} \ln \frac{1}{N} W$ and

$$
W=\max \left\{\frac{\min _{j} \sum_{k=1}^{K} \mu_{k, j} Q_{k}^{S T}}{\min _{m, i} \mu_{m, i} \bar{I}_{i, s^{m} \mid p}}, \frac{\min _{j, l} \sum_{k=1}^{K}\left(\mu_{k, j}+\mu_{k, l}\right) Q_{k}^{S T}}{\max _{m, i} \mu_{m, i} \bar{I}_{i, s^{m} \mid p}}\right\}
$$

the duality gap approaches 0 as $\left(1-d a b \frac{1}{\left(e^{c^{\prime} \varepsilon T}+a\right)\left(e^{c^{\prime} \varepsilon T}+b\right)}\right){ }^{M N^{2} T}$, or equivalently $\left(1-e^{c^{\prime 2} \varepsilon^{2} T^{2}}\right)^{M N^{2} T}$, for $T \rightarrow \infty$.

\section{REFERENCES}

[1] B. Wang and K. J. R. Liu, "Advances in cognitive radio networks: A survey," IEEE J. Sel. Topics Signal Process., vol. 5, no. 1, pp. 5-23, Feb. 2011.

[2] S. Haykin, "Cognitive radio: Brain-empowered wireless communications," IEEE J. Sel. Areas Commun., vol. 23, no. 2, pp. 201-220, Feb. 2005.

[3] M. G. Khoshkholgh, K. Navaie, and H. Yanikomeroglu, "Access strategies for spectrum sharing in fading environment," IEEE Trans. Mobile Comput., vol. 9, no. 12, pp. 1780-1793, Dec. 2010.

[4] G. Bansal, M. J. Hossain, V. K. Bhargava, and T. Le-Ngoc, "Subcarrier and power allocation for OFDMA-based cognitive radio systems with joint overlay and underlay spectrum access mechanism," IEEE Trans. Veh. Technol., vol. 62, no. 3, pp. 1111-1122, Mar. 2013.

[5] R. Zhang, Y-C. Liang, and S. Cui, "Dynamic resource allocation in cognitive radio networks: A convex optimization perspective," IEEE Signal Process. Mag., vol. 27, no. 3, pp. 102-114, May 2010.

[6] B. Kaufman, J. Lilleberg, and B. Aazhang, "Spectrum sharing scheme between cellular users and ad-hoc device-to-device users," IEEE Trans. Wireless Commun., vol. 12, no. 3, pp. 1038-1049, Mar. 2013.

[7] V. Chandrasekhar, J. G. Andrews, and A. Gatherer, "Femtocell networks: A survey," IEEE Commun. Mag., vol. 46, no. 9, pp. 59-67, Sep. 2008.

[8] L. Li, C. Xu, and M. Tao, "Resource allocation in open access OFDMA femtocell networks," IEEE Wireless Commun. Lett., vol. 1, no. 6, pp. 625-628, Dec. 2012.

[9] R. Zhang, S. Cui, and Y-C. Liang, "On ergodic sum capacity of fading cognitive multiple-access and broadcast channels," IEEE Trans. Inf. Theory, vol. 55, no. 11, pp. 5161-5178, Nov. 2009.

[10] X. Kang, Y-C. Kiang, and H. K. Garg, "Fading cognitive multiple access channels: Outage capacity regions and optimal power allocation," IEEE Trans. Wireless Commun., vol. 9, no. 7, pp. 2382-2391, Jul. 2010.

[11] V. Asghari and S. Aissa, "Resource management in spectrum-sharing cognitive radio broadcast channels: Adaptive time and power allocation," IEEE Trans. Commun, vol. 59, no. 5, pp. 1446-1457, May 2011.

[12] D. T. Ngo, C. Tellambura, and H. H. Nguyen, "Resource allocation for OFDMA-based cognitive radio multicast networks with primary user activity consideration," IEEE Trans. Veh. Technol., vol. 59, no. 4, pp. 1668-1679, May 2010.

[13] M. Naeem, A. S. Khwaja, A. Anpalagan, and M. Jaseemuddin, "Green cooperative cognitive radio: A multiobjective optimization paradigm," IEEE Syst. J., to be published.

[14] R. Devarajan, S. C. Jha, U. Phuyal, and V. K. Bhargava, "Energy-aware resource allocation for cooperative cellular network using multi-objective optimization approach," IEEE Trans. Wireless Commun., vol. 11, no. 5, pp. 1797-1807, May 2014.

[15] E. Bedeer, O. Dobre, M. Ahmed, and K. Baddour, "A multiobjective optimization approach for optimal link adaptation of OFDM based cognitive radio systems with imperfect spectrum sensing," IEEE Trans. Wireless Commun., vol. 13, no. 4, pp. 2339-2351, Apr. 2014.

[16] S. Rangan and R. Madan, "Belief propagation methods for intercell interference coordination in femtocell networks," IEEE J. Sel. Areas Commun., vol. 30, no. 3, pp. 631-640, Apr. 2014.

[17] M. Shamaiah, S. H. Lee, S. Vishwanath, and H. Vikalo, "Distributed algorithms for spectrum access in cognitive radio relay networks," IEEE J. Sel. Areas Commun., vol. 30, no. 10, pp. 1947-1957, Nov. 2012.

[18] X. Gong, S. A. Vorobyov, and C. Tellambura, "Optimal bandwidth and power allocation for sum ergodic capacity under fading channels in 
cognitive radio networks," IEEE Trans. Signal Process., vol. 59, no. 4, pp. 1814-1826, Apr. 2011.

[19] S. Ekin, M. M. Abdallah, K. A. Qaraqe, and E. Serpedin, "Random subcarrier allocation in OFDM-based cognitive radio networks," IEEE Trans. Signal Process., vol. 60, no. 9, pp. 4758-4774, Sep. 2012.

[20] Y. Zhang and C. Leung, "An efficient power-loading scheme for OFDMbased cognitive radio systems," IEEE Trans. Veh. Technol., vol. 59, no. 4, pp. 1858-1864, May 2010.

[21] S. M. Almalfouh and G. L. Stuber, "Interference-aware radio resource allocation in OFDMA-based cognitive radio networks," IEEE Trans. Veh. Technol., vol. 60, no. 4, pp. 1699-1713, May 2011.

[22] A. M-C. So and Y. J. Zhang, "Distributionally robust slow adaptive OFDMA with soft QoS via linear programming," IEEE J. Sel. Areas Commun., vol. 31, no. 5, pp. 947-958, May 2013.

[23] M. Ge and S. Wang, "Fast optimal resource allocation is possible for multiuser OFDM-based cognitive radio networks with heterogeneous services," IEEE Trans. Wireless Commun., vol. 11, no. 4, pp. 1500-1509, Apr. 2012.

[24] A. G. Marques, L. M. Lopez-Ramos, G. B. Giannakis, and J. Ramos, "Resource allocation for interweave and underlay CRs under probabilityof-interference constraints," IEEE J. Sel. Areas Commun., vol. 30, no. 10, pp. 1922-1933, Nov. 2012.

[25] N. Y. Soltani, S-J. Kim, and G. B. Giannakis, "Chance-constrained optimization of OFDMA cognitive radio uplinks," IEEE Trans. Wireless Commun., vol. 12, no. 3, pp. 1098-1107, Mar. 2013.

[26] M. Karaca, K. Khalil, E. Ekici, and O. Ercetin, "Optimal scheduling and power allocation in cooperate-to-join cognitive radio networks," IEEE/ACM Trans. Netw., vol. 21, no. 6, pp. 1708-1721, Dec. 2013.

[27] C-H. Chen, C-L. Wang, and C-T. Chen, "A resource allocation scheme for cooperative multiuser OFDM-based cognitive radio systems," IEEE Trans. Commun., vol. 59, no. 11, pp. 3204-3215, Nov. 2011.

[28] H. Xu and B. Li, "Resource allocation with flexible channel cooperation in cognitive radio networks," IEEE Trans. Mobile Comput., vol. 12, no. 5, pp. 957-970, May 2013

[29] R. Urgaonkar and M. J. Neely, "Opportunistic cooperation in cognitive femtocell networks," IEEE J. Sel. Areas Commun., vol. 30, no. 3, pp. 607-616, Apr. 2012.

[30] S. Bayhan and F. Alagoz, "Scheduling in centralized cognitive radio networks for energy efficiency," IEEE Trans. Veh. Technol., vol. 62, no. 2, pp. 582-595, Feb. 2013

[31] K. W. Choi, E. Hossain, and D. I. Kim, "Downlink subchannel and power allocation in multi-cell OFDMA cognitive radio networks," IEEE Trans. Wireless Commun., vol. 10, no. 7, pp. 2259-2271, Jul. 2011.

[32] S. Boyd and L. Vandenberghe, Convex optimization. Cambridge, U.K.: Cambridge Univ. Press, 2004.

[33] W. Yu and R. Lui, "Dual methods for nonconvex spectrum optimization of multicarrier systems," IEEE Trans. Commun., vol. 54, no. 7, pp. 1310-1322, Jul. 2006

[34] Z. Q. Luo and S. Zhang, "Dynamic spectrum management: Complexity an duality," IEEE J. Sel. Topics Signal Process., vol. 2, no. 1, pp. 57-73, Feb. 2008.

[35] Z. Q. Luo and S. Zhang, "Duality gap estimation and polynominal time approximation for optimal spectrum management," IEEE Trans. Signal Process., vol. 57, no. 7, pp. 2675-2689, Jul. 2009.

[36] P. Tsiaflakis, I. Necoara, J. A. K. Suykens, and M. Moonen, "Improved dual decomposition based optimization for DSL dynamic spectrum management," IEEE Trans. Signal Process., vol. 58, no. 4, pp. 2230-2245, Apr. 2010.

[37] K. Seong, M. Mohseni, and J. Cioffi, "Optimal resource allocation for OFDMA downlink systems," in Proc. IEEE Int. Symp. Inf. Theory, Jul. 9-14, 2006, pp. 1394-1398.

[38] I. C. Wong and B. L. Evans, "Optimal downlink OFDMA resource allocation with linear complexity to maximize ergodic capacity," IEEE Trans. Wireless Commun., vol. 7, no. 3, pp. 962-971, Mar. 2008.

[39] B. G. Kim and J. W. Lee, "Joint opportunistic subchannel and power with scheduling at relay stations," IEEE Trans. Veh. Technol., vol. 59, no. 5, pp. 2138-2148, Jun. 2010.

[40] M. G. Khoshkholgh, K. Navaie, and H. Yanikomeroglu, "Achievable capacity in hybrid DS-CDMA/OFDM spectrum-sharing," IEEE Trans. Mobile Comput., vol. 9, no. 6, pp. 756-777, Jun. 2010.

[41] A. Ghasemi and E. S. Sousa, "Fundamental limits of spectrum-sharing in fading environments," IEEE Trans. Wireless Commun., vol. 6, no. 2, pp. 649-658, Feb. 2007.

[42] R. Zhang, "On peak versus average interference power constraints for protecting primary users in cognitive radio networks," IEEE Trans. Wireless Commun., vol. 8, no. 4, pp. 2112-2120, Apr. 2009.
[43] R. Etkin, A. Parekh, and D. N. C. Tse, "Spectrum sharing in unlicensed bands," IEEE J. Sel. Areas Commun., vol. 25, no. 3, pp. 517-528, Apr. 2007.

[44] J. Jang and K. B. Lee, "Transmit power adaptation for multiuser OFDM systems," IEEE J. Sel. Areas Commun., vol. 21, no. 2, pp. 171-178, Feb. 2003.

[45] H. Liu and G. Li, OFDM-Based Broadband Wireless Networks: Design and Optimization. Hoboken, NJ, USA: Wiley.

[46] R. Etkin and D. N. C. Tse, "Gaussian interference channel capacity to within one bit," IEEE Trans. Inf. Theory, vol. 54, no. 12, pp. 5534-5562, Dec. 2008.

[47] C. Suh and D. N. C. Tse, "Feedback capacity of the Gaussian interference channel to within 2 bits," IEEE Trans. Inf. Theory, vol. 57, no. 5, pp. 2667-2685, May 2011.

[48] X. Wang and N. Gao, "Stochastic resource allocation over fading multiple access and broadcast channels," IEEE Trans. Inf. Theory, vol. 56, no. 5 , pp. 2382-2391, May 2010.

[49] X. Wang and G. B. Giannakis, "Resource allocation for wireless multiuser OFDM networks," IEEE Trans. Inf. Theory, vol. 57, no. 7, pp. 4359-4372, Jul. 2011.

[50] D. T. Ngo, S. Khakurel, and T. L. Ngoc, "Joint subchannel assignment and power allocation for OFDMA femtocell networks," IEEE Trans. Wireless Commun., vol. 13, no. 1, pp. 342-355, Jul. 2013.

[51] D. P. Bertsekas, Constrained Optimization and Lagrange Multiplier Method. New York, NY, USA: Academic, 1982.

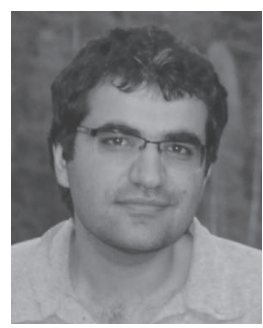

Mohammad G. Khoshkholgh received the B.Sc. degree in electrical engineering from Isfahan University, Isfahan, Iran, in 2006 and the M.Sc. degree in electrical engineering from Tarbiat Modares University, Tehran, Iran, in 2008. From 2008 until 2012, he was with the Wireless Innovation Laboratory, Tarbiat Modares University. From February 2012 to February 2014, he was with Simula Research Laboratory, Fornebu, Norway, working on developing communication solutions for smart grid systems. He is currently with the University of British Columbia. His research interests are mainly in wireless communications, radio resource allocations, and spectrum sharing.

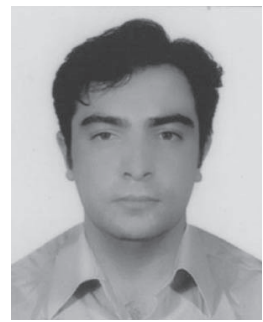

Nader Mokari Yamchi is currently working toward the Ph.D. degree with Tarbiat Modares University, Tehran, Iran. His research interests include wireless communications, radio resource allocations, cooperative, network planning, and spectrum sharing. 


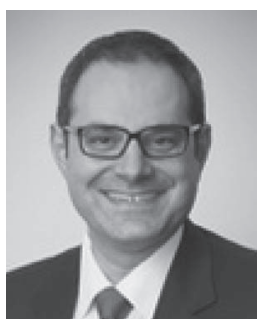

Keivan Navaie (SM'10) received the B.Sc. degree from Sharif University of Technology, Tehran, Iran, in 1995, the M.Sc. degree from the University of Tehran, Tehran, in 1997, and the Ph.D. degree from Tarbiat Modares University, Tehran, in 2004, all in electrical engineering. From March to November 2004, he was a Postdoctoral Research Fellow with the School of Mathematics and Statistics, Carleton University, Ottawa, Canada. From December 2004 to September 2006, he was with the Broadband Communications and Wireless System (BCWS) Centre, Carleton University, where he was the Project Manager of BCWS participation in European Union Sixth Framework integrated project, the Wireless World Initiative New Radio (WINNER) on beyond 3G wireless systems. From September 2006 to July 2011, he was with the Department of Electrical and Computer Engineering, Tarbiat Modares University. Since July 2011, he has been with the School of Electronic and Electrical Engineering, University of Leeds, Leeds, U.K. His research interests lie in the field of radio resource allocation for wireless communication systems, dynamic spectrum allocation, cognitive radio networks, and cooperative communications. He has been on the technical program committee of different IEEE conferences, including GlobeCom, ICC, VTC, and WCNC, and chaired some of their symposia. He also served as a Cochair of the Wireless Network Track, IEEE VTC 2012, Yokohama, Japan, and the IEEE Eighth International Workshop on Wireless Network Measurements (WiNMee) 2012, Paderborn, Germany. He is on the editorial board of the European Transactions on Telecommunications. He was a recipient of the 2011 IEEE Iran Section Young Investigator Award.

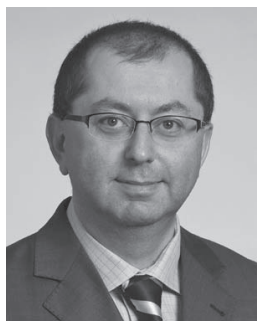

Halim Yanikomeroglu (S'96-M'98-SM'12) received the B.Sc. degree in electrical and electronics engineering from Middle East Technical University, Ankara, Turkey, in 1990 and the M.A.Sc. degree in electrical engineering and the Ph.D. degree in electrical and computer engineering from the University of Toronto, Toronto, Canada, in 1992 and 1998, respectively. From 1993 to 1994, he was with the R\&D Group, Marconi Komunikasyon A.S., Ankara. Since 1998, he has been with the Department of Systems and Computer Engineering, Carleton University, Ottawa, Canada, where he is currently a Full Professor. He is also an Adjunct Professor with the Advanced Technology Research Institute, King Saud University, Riyadh, Saudi Arabia. During the 2011-2012 academic year, he was a Visiting Professor at TOBB University of Economics and Technology, Ankara. His research interests cover many aspects of the physical, medium access, and networking layers of wireless communications, with a special emphasis on the next-generation cellular system. In recent years, his research has been funded by Huawei (Canada and China), Research In Motion (Canada), Samsung Advanced Institute of Technology (Korea), Communications Research Centre of Canada, Nortel, Natural Sciences and Engineering Research Council of Canada, and Carleton University. He is a Distinguished Lecturer of the IEEE Vehicular Technology Society. He has been involved in the steering committees and technical program committees of numerous international conferences in communications. He has also given about 20 tutorials in such conferences. He is a member of the steering committee of the IEEE Wireless Communications and Networking Conference (WCNC) and has been involved in the organization of this conference over the years, including serving as the Technical Program Cochair of WCNC 2004 and the Technical Program Chair of WCNC 2008. He also served as the Technical Program Cochair of WCNC 2014 held in Istanbul. He was the General Cochair of the IEEE Vehicular Technology Conference Fall 2010 held in Ottawa. He was also the Chair of the IEEE Technical Committee on Personal Communications (now Wireless Technical Committee). He is currently an Editor of the IEEE TRANSACTIONS ON COMMUNICATIONS. He was an Editor of the IEEE TRANSACTIONS ON WIRELESS COMmUniCATIONS and IEEE Communications Surveys and Tutorials. He was a recipient of the Carleton University Faculty Graduate Mentoring Award in 2010, the Carleton University Graduate Students Association Excellence Award in Graduate Teaching in 2010, and the Carleton University Research Achievement Award in 2009. He is a Registered Professional Engineer in the Province of Ontario, Canada.

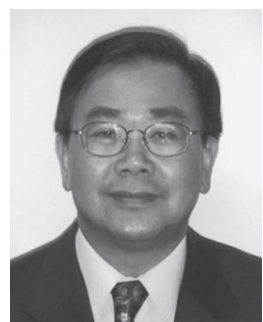

Victor C. M. Leung (S'75-M'89-SM'97-F'03) received the B.A.Sc.(Hons.) and the Ph.D. (on a Natural Sciences and Engineering Research Council Postgraduate Scholarship) degrees in electrical engineering from The University of British Columbia (UBC), Vancouver, Canada, in 1977 in 1981, respectively.

From 1981 to 1987, he was a Senior Member of Technical Staff and a Satellite System Specialist at MPR Teltech Ltd., Burnaby, Canada. In 1988, he was a Lecturer with the Department of Electronics, The Chinese University of Hong Kong. In 1989, he rejoined, as a Faculty Member, UBC, where he is currently a Professor and the TELUS Mobility Research Chair in Advanced Telecommunications Engineering in the Department of Electrical and Computer Engineering. He has coauthored over 700 technical papers in international journals and conference proceedings and 29 book chapters and has coedited eight book titles. Several of his papers had been selected for best paper awards. His research interests are in the areas wireless networks and mobile systems.

Dr. Leung is a Fellow of The Royal Society of Canada, The Engineering Institute of Canada, and The Canadian Academy of Engineering. He was a Distinguished Lecturer of the IEEE Communications Society. $\mathrm{He}$ is a member of the editorial boards of the IEEE WIRELESS COMMUNICATIONS LETTERs, Computer Communications, and several other journals. He has previously served on the editorial boards of the IEEE JOURNAL ON SELECTED AREAS IN COMMUNICATIONS-Wireless Communications Series, the IEEE TRANSACTIONS ON Wireless COMMUNiCATIONS, the IEEE TRANSACTIONS ON VEHICULAR TECHNOLOGY, the IEEE TRANSACTIONS ON COMPUTERs, and the Journal of Communications and Networks. He has been a Guest Editor of many journal special issues and has contributed to the organizing committees and technical program committees of numerous conferences and workshops. He was a recipient of the APEBC Gold Medal as the head of the graduating class in the Faculty of Applied Science during his B.A.Sc. studies at UBC. He was also a recipient of the IEEE Vancouver Section Centennial Award and the 2012 UBC Killam Research Prize. He is a Registered Professional Engineer in the Province of British Columbia, Canada.

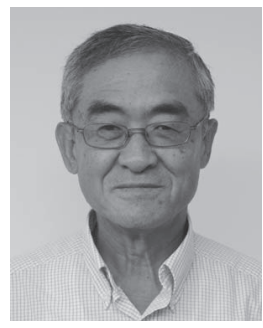

Kang G. Shin (LF'12) is currently the Kevin and Nancy O'Connor Professor of Computer Science with the Department of Electrical Engineering and Computer Science, University of Michigan, Ann Arbor, MI, USA. He has been a Cofounder of a couple of start-ups and has also licensed some of his technologies to industry. He has supervised the completion of 74 Ph.D. studies. He has authored/ coauthored over 800 technical articles (more than 300 of these are in archival journals), a textbook, and over 20 patents or invention disclosures. His current research focuses on QoS-sensitive computing and networking and on embedded real-time and cyberphysical systems.

Prof. Shin received numerous best paper awards, including the Best Paper Awards from the 2011 ACM International Conference on Mobile Computing and Networking (MobiCom'11), the 2011 IEEE International Conference on Autonomic Computing, and the 2010 and 2000 USENIX Annual Technical Conferences, the 2003 IEEE Communications Society William R. Bennett Prize Paper Award, and the 1987 Outstanding IEEE Transactions on Automatic Control Paper Award. He has also received several institutional awards, including the Research Excellence Award in 1989, the Outstanding Achievement Award in 1999, the Distinguished Faculty Achievement Award in 2001, and the Stephen Attwood Award in 2004 from the University of Michigan (the highest honor bestowed to Michigan Engineering faculty); a Distinguished Alumni Award of the College of Engineering, Seoul National University in 2002; the 2003 IEEE RTC Technical Achievement Award; and the 2006 Ho-Am Prize in Engineering (the highest honor bestowed to Korean-origin engineers). 